\title{
Difluoromethyltrialkylammonium salts - their expeditious synthesis from chlorodifluoromethane and tertiary amines in the presence of concentrated aqueous sodium hydroxide. The catalytic process.
}

\author{
Ewelina Nawrot, Andrzej Jończyk
}

\section{Supporting Information Available:}

(1) compound characterization data $\left({ }^{1} \mathrm{H}\right.$ NMR, ${ }^{13} \mathrm{C} \quad \mathrm{NMR},{ }^{19} \mathrm{~F} \quad \mathrm{NMR}$ and elemental analysis or HRMS of $2 \mathbf{a}-\mathrm{g}, 3 \mathbf{h}-\mathbf{l}, 4 \mathbf{h}-\mathbf{k}, 5 \mathbf{i}, 5 \mathbf{j}$ )

page S1-S3

(2) copies of ${ }^{1} \mathrm{H}$ NMR spectra of compounds: $\mathbf{2 a}$ - page S4, 2b - page S5, 2c - page S6, 2d - page S7, 2e - page S8, 2f page S9, 2g - page S10, 3h - page S11, 3i - page S12, 3j - page S13, 3k - page S14, 31 - page S15, 4h - page S16, 4i page $S 17, \mathbf{4 j}$ - page $S 18, \mathbf{4 k}$ - page $S 19, \mathbf{5 i}$ - page S20, $\mathbf{5 j}$ - page $S 21$

2a, $N$-decyl- $N$-difluoromethylpiperidineammonium chloride, m.p. $135-137^{\circ} \mathrm{C} ;{ }^{1} \mathrm{H}$ NMR $\left(400 \mathrm{MHz}, \mathrm{CDCl}_{3}, 25^{\circ} \mathrm{C}\right): \delta=0.75$ $\left(\mathrm{t},{ }^{3} \mathrm{~J}(\mathrm{H}, \mathrm{H})=6.8 \mathrm{~Hz}, 3 \mathrm{H}, \mathrm{CH}_{3}\right), 1.13-1.25\left(\mathrm{~m}, 14 \mathrm{H}, 7 \mathrm{xCH}_{2}\right), 1.70-1.96\left(\mathrm{~m}, 8 \mathrm{H}, 4 \mathrm{xCH}_{2}\right), 3.58-3.66\left(\mathrm{~m}, 4 \mathrm{H}, 2 \mathrm{xCH}_{2}\right), 4.06-4.12$ $\left(\mathrm{m}, 2 \mathrm{H}, \mathrm{CH}_{2}\right), 8.41\left(\mathrm{t},{ }^{3} \mathrm{~J}(\mathrm{~F}, \mathrm{H})=58 \mathrm{~Hz}, 1 \mathrm{H}, \mathrm{CHF}_{2}\right) ;{ }^{13} \mathrm{C} \mathrm{NMR}\left(100 \mathrm{MHz}, \mathrm{CDCl}_{3}, 25^{\circ} \mathrm{C}\right): \delta=13.8,19.2,20.0,22.4,22.9,26.5$, $28.7,28.9,29.0,29.1,31.5,54.2,54.5,115.4\left(\mathrm{t}, J=276 \mathrm{~Hz}, \mathrm{CHF}_{2}\right) ;{ }^{19} \mathrm{~F}$ NMR $\left(376 \mathrm{MHz}, \mathrm{CDCl}_{3}, 25^{\circ} \mathrm{C}\right): \delta=-118.42(\mathrm{~d}, J=59$ $\mathrm{Hz}, 2 \mathrm{~F}$ ); Anal. calcd. for $\mathrm{C}_{16} \mathrm{H}_{32} \mathrm{NF}_{2} \mathrm{Cl}$ : C, 61.62, H, 10.34, N, 4.49. Found: C, 61.69, H, 10.25, N, 4.56.

2b, $N$-decyl- $N$-difluoromethyl- $N$-ethyl- $N$-methylammonium chloride, ${ }^{1} \mathrm{H}$ NMR $\left(400 \mathrm{MHz}, \mathrm{CDCl}_{3}, 25^{\circ} \mathrm{C}\right): \delta=0.77$ (t, $\left.{ }^{3} J(\mathrm{H}, \mathrm{H})=6.6 \mathrm{~Hz}, 3 \mathrm{H}, \mathrm{CH}_{3}\right), 1.15-1.30\left(\mathrm{~m}, 14 \mathrm{H}, 7 \mathrm{xCH}_{2}\right), 1.42\left(\mathrm{t},{ }^{3} J(\mathrm{H}, \mathrm{H})=7.2 \mathrm{~Hz}, 3 \mathrm{H}, \mathrm{CH}_{3}\right), 1.72-1.77\left(\mathrm{~m}, 2 \mathrm{H}, \mathrm{CH}_{2}\right), 3.39(\mathrm{~s}$, $\left.3 \mathrm{H}, \mathrm{CH}_{3}\right), 3.57-3.70\left(\mathrm{~m}, 2 \mathrm{H}, \mathrm{CH}_{2}\right), 3.80-3.94\left(\mathrm{~m}, 2 \mathrm{H}, \mathrm{CH}_{2}\right), 8.39\left(\mathrm{t},{ }^{3} \mathrm{~J}(\mathrm{~F}, \mathrm{H})=58 \mathrm{~Hz}, 1 \mathrm{H}, \mathrm{CHF}_{2}\right) ;{ }^{13} \mathrm{C} \mathrm{NMR}\left(100 \mathrm{MHz} \mathrm{CDCl}_{3}\right.$, $\left.25^{\circ} \mathrm{C}\right): \delta=8.6,13.9,22.4,22.5,26.3,28.8,29.0,29.1,31.6,43.4,54.7,58.2,114.8\left(\mathrm{t}, J=275 \mathrm{~Hz}, \mathrm{CHF}_{2}\right) ;{ }^{19} \mathrm{~F}$ NMR $(376$ $\left.\mathrm{MHz}, \mathrm{CDCl}_{3}, 25^{\circ} \mathrm{C}\right): \delta=-117.31(\mathrm{~d}, J=58 \mathrm{~Hz}, 2 \mathrm{~F})$; HRMS calcd. for $\left[\mathrm{C}_{14} \mathrm{H}_{30} \mathrm{~F}_{2} \mathrm{~N}\right]^{+}: 250.23408$. Found: 250.23296.

2c, $N, N$-dibuthyl- $N$-difluoromethyl- $N$-phenethylammonium chloride, m.p. $96-98^{\circ} \mathrm{C} ;{ }^{1} \mathrm{H}$ NMR $\left(400 \mathrm{MHz}, \mathrm{CDCl}_{3}, 25^{\circ} \mathrm{C}\right)$ : $\delta=0.93\left(\mathrm{t},{ }^{3} \mathrm{~J}(\mathrm{H}, \mathrm{H})=7.4 \mathrm{~Hz}, 6 \mathrm{H}, 2 \mathrm{xCH}_{3}\right), 1.32-1.41\left(\mathrm{~m}, 4 \mathrm{H}, 2 \mathrm{xCH}_{2}\right), 1.74-1.82\left(\mathrm{~m}, 4 \mathrm{H}, 2 \mathrm{xCH}_{2}\right), 3.15-3.19\left(\mathrm{~m}, 2 \mathrm{H}, \mathrm{CH}_{2}\right)$, 3.78-3.82 (m, 4H, $\left.2 \mathrm{xCH}_{2}\right), 3.87-3.91\left(\mathrm{~m}, 2 \mathrm{H}, \mathrm{CH}_{2}\right), 7.21-7.30(\mathrm{~m}, 5 \mathrm{H}, \mathrm{ArH}), 8.82\left(\mathrm{t},{ }^{3} \mathrm{~J}(\mathrm{~F}, \mathrm{H})=58 \mathrm{~Hz}, 1 \mathrm{H}, \mathrm{CHF}_{2}\right) ;{ }^{13} \mathrm{C} \mathrm{NMR}^{1}$ $\left(100 \mathrm{MHz}, \mathrm{CDCl}_{3}, 25^{\circ} \mathrm{C}\right): \delta=13.4,19.8,24.6,29.3,57.5,58.3,115.3\left(\mathrm{t}, J=276 \mathrm{~Hz}, \mathrm{CHF}_{2}\right), 127.5,128.9,129.0,134.7 ;{ }^{19} \mathrm{~F}$ NMR $\left(376 \mathrm{MHz}, \mathrm{CDCl}_{3}, 25^{\circ} \mathrm{C}\right): \delta=-113.71(\mathrm{~d}, J=58 \mathrm{~Hz}, 2 \mathrm{~F})$; Anal. calcd. for $\mathrm{C}_{17} \mathrm{H}_{28} \mathrm{NF}_{2} \mathrm{Cl}$ : C, 63.84, H, 8.82, N, 4.38. Found: C, 63.88, H, 8.61, N, 4.46 .

2d, $N$-difluoromethyl- $N, N, N$-trioctylammonium chloride, m.p. $88-89^{\circ} \mathrm{C} ;{ }^{1} \mathrm{H}$ NMR $\left(400 \mathrm{MHz}, \mathrm{CDCl}_{3}, 25^{\circ} \mathrm{C}\right): \delta=0.77$ (t, $\left.{ }^{3} J(\mathrm{H}, \mathrm{H})=6.8 \mathrm{~Hz}, 9 \mathrm{H}, 3 \mathrm{xCH}_{3}\right), 1.10-1.30\left(\mathrm{~m}, 30 \mathrm{H}, 15 \mathrm{xCH}_{2}\right), 1.68-1.78\left(\mathrm{~m}, 6 \mathrm{H}, 3 \mathrm{xCH}_{2}\right), 3.60-3.64\left(\mathrm{~m}, 6 \mathrm{H}, 3 \mathrm{xCH}_{2}\right), 8.61(\mathrm{t}$, $\left.{ }^{3} J(\mathrm{~F}, \mathrm{H})=58 \mathrm{~Hz}, 1 \mathrm{H}, \mathrm{CHF}_{2}\right) ;{ }^{13} \mathrm{C} \mathrm{NMR}\left(100 \mathrm{MHz}, \mathrm{CDCl}_{3}, 25^{\circ} \mathrm{C}\right): \delta=13.8,22.3,22.6,26.4,28.7,28.8,31.4,57.3,115.2(\mathrm{t}, J=$ $\left.276 \mathrm{~Hz}, \mathrm{CHF}_{2}\right) ;{ }^{19} \mathrm{~F}$ NMR $\left(376 \mathrm{MHz}, \mathrm{CDCl}_{3}, 25^{\circ} \mathrm{C}\right): \delta=-113.90(\mathrm{~d}, J=58 \mathrm{~Hz}, 2 \mathrm{~F})$; Anal. calcd. for $\mathrm{C}_{25} \mathrm{H}_{52} \mathrm{NF}_{2} \mathrm{Cl}: \mathrm{C}, 68.22$, $\mathrm{H}, 11.91, \mathrm{~N}, 3.18$. Found: C, 68.23, H, 11.92, N, 3.23.

2e, $N, N, N$-tributhyl- $N$-difluoromethylammonium chloride, m.p. $91-93{ }^{\circ} \mathrm{C} ;{ }^{1} \mathrm{H}$ NMR $\left(400 \mathrm{MHz}, \mathrm{CDCl}_{3}, 25^{\circ} \mathrm{C}\right): \delta=0.87$ (t, $\left.{ }^{3} J(\mathrm{H}, \mathrm{H})=7.4 \mathrm{~Hz}, 9 \mathrm{H}, 3 \mathrm{xCH}_{3}\right), 1.26-1.36\left(\mathrm{~m}, 6 \mathrm{H}, 3 \mathrm{xCH}_{2}\right), 1.67-1.75\left(\mathrm{~m}, 6 \mathrm{H}, 3 \mathrm{xCH}_{2}\right), 3.62-3.66\left(\mathrm{~m}, 6 \mathrm{H}, 3 \mathrm{xCH}_{2}\right), 8.51(\mathrm{t}$, $\left.{ }^{3} J(\mathrm{~F}, \mathrm{H})=58 \mathrm{~Hz}, 1 \mathrm{H}, \mathrm{CHF}_{2}\right) ;{ }^{13} \mathrm{C} \mathrm{NMR}\left(100 \mathrm{MHz}, \mathrm{CDCl}_{3}, 25^{\circ} \mathrm{C}\right): \delta=13.2,19.7,24.4,57.3,115.1\left(\mathrm{t}, J=276 \mathrm{~Hz}, \mathrm{CHF}_{2}\right) ;{ }^{19} \mathrm{~F}$ NMR $\left(376 \mathrm{MHz}, \mathrm{CDCl}_{3}, 25^{\circ} \mathrm{C}\right): \delta=-114.33(\mathrm{~d}, J=58 \mathrm{~Hz}, 2 \mathrm{~F})$; Anal. calcd. for $\mathrm{C}_{13} \mathrm{H}_{28} \mathrm{NF}_{2} \mathrm{Cl}$ : C, 57.44, H, 10.38, N, 5.15, Cl, 13.04. Found: C, 57.25, H, 10.24, N, 5.22, Cl, 12.99 .

2f, $N$-difluoromethyl- $N$-hexadecyl- $N$-dimethylammonium chloride, m.p. $97-100^{\circ} \mathrm{C} ;{ }^{1} \mathrm{H} \mathrm{NMR}\left(400 \mathrm{MHz}, \mathrm{CDCl}_{3}, 25^{\circ} \mathrm{C}\right)$ : $\delta=0.80\left(\mathrm{t},{ }^{3} J(\mathrm{H}, \mathrm{H})=6.8 \mathrm{~Hz}, 3 \mathrm{H}, \mathrm{CH}_{3}\right), 1.17-1.28\left(\mathrm{~m}, 26 \mathrm{H}, 13 \mathrm{xCH}_{2}\right), 1.74-1.86\left(\mathrm{~m}, 2 \mathrm{H}, \mathrm{CH}_{2}\right), 3.48\left(\mathrm{~s}, 6 \mathrm{H}, 2 \mathrm{xCH}_{3}\right), 3.72-3.76$ $\left(\mathrm{m}, 2 \mathrm{H}, \mathrm{CH}_{2}\right), 8.38\left(\mathrm{t},{ }^{3} \mathrm{~J}(\mathrm{~F}, \mathrm{H})=59 \mathrm{~Hz}, 1 \mathrm{H}, \mathrm{CHF}_{2}\right) ;{ }^{13} \mathrm{C} \mathrm{NMR}\left(100 \mathrm{MHz}, \mathrm{CDCl}_{3}, 25^{\circ} \mathrm{C}\right): \delta=14.0,22.5,26.3,28.9,29.2,29.3$, 29.4, 29.48, 29.50, 29.51, 31.7, 45.3, 60.8, 114.4 (t, $\left.J=275 \mathrm{~Hz}, \mathrm{CHF}_{2}\right) ;{ }^{19} \mathrm{~F}$ NMR $\left(376 \mathrm{MHz}, \mathrm{CDCl}_{3}, 25^{\circ} \mathrm{C}\right): \delta=-110.18(\mathrm{~d}, J$ $=58 \mathrm{~Hz}, 2 \mathrm{~F})$; HRMS calcd. for $\left[\mathrm{C}_{19} \mathrm{H}_{40} \mathrm{NF}_{2}\right]^{+}: 320.31233$. Found: 320.31377 .

2g, $N$-difluoromethyl- $N, N, N$-tripropylammonium chloride, m.p. $111-113^{\circ} \mathrm{C}$ decomp.; ${ }^{1} \mathrm{H}$ NMR $\left(400 \mathrm{MHz}, \mathrm{CDCl}_{3}, 25^{\circ} \mathrm{C}\right)$ : $\delta=0.91\left(\mathrm{t},{ }^{3} J(\mathrm{H}, \mathrm{H})=7.2 \mathrm{~Hz}, 9 \mathrm{H}, 3 \mathrm{xCH}_{3}\right), 1.74-1.83\left(\mathrm{~m}, 6 \mathrm{H}, 3 \mathrm{xCH}_{2}\right), 3.57-3.62\left(\mathrm{~m}, 6 \mathrm{H}, 3 \mathrm{xCH}_{2}\right), 8.50\left(\mathrm{t},{ }^{3} J(\mathrm{~F}, \mathrm{H})=58 \mathrm{~Hz}, 1 \mathrm{H}\right.$, $\left.\mathrm{CHF}_{2}\right) ;{ }^{13} \mathrm{C} \mathrm{NMR}\left(100 \mathrm{MHz}, \mathrm{CDCl}_{3}, 2^{\circ} \mathrm{C}\right): \delta=10.7,16.2,58.7,115.0\left(\mathrm{t}, J=276 \mathrm{~Hz}, \mathrm{CHF}_{2}\right) ;{ }^{19} \mathrm{~F} \mathrm{NMR}\left(376 \mathrm{MHz}^{\circ} \mathrm{CDCl}_{3}\right.$, $25^{\circ} \mathrm{C}$ ): $\delta=-114.84(\mathrm{~d}, J=58 \mathrm{~Hz}, 2 \mathrm{~F})$; Anal. calcd. for $\mathrm{C}_{10} \mathrm{H}_{22} \mathrm{NF}_{2} \mathrm{Cl}: \mathrm{C}, 52.28, \mathrm{H}, 9.65, \mathrm{~N}, 6.10$. Found: C, 52.10, H, 9.76, N, 5.94 . 
3h, $N$-buthyl- $N$-difluoromethyl- $N$-ethyl- $N$-methylammonium iodide, m.p. $94-96{ }^{\circ} \mathrm{C}$ decomp.; ${ }^{1} \mathrm{H}$ NMR $\left(400 \mathrm{MHz}, \mathrm{CDCl}_{3}\right.$, $\left.25^{\circ} \mathrm{C}\right): \delta=0.95\left(\mathrm{t},{ }^{3} J(\mathrm{H}, \mathrm{H})=7.4 \mathrm{~Hz}, 3 \mathrm{H}, \mathrm{CH}_{3}\right), 1.35-1.43\left(\mathrm{~m}, 2 \mathrm{H}, \mathrm{CH}_{2}\right), 1.48\left(\mathrm{t},{ }^{3} \mathrm{~J}(\mathrm{H}, \mathrm{H})=7.2 \mathrm{~Hz}, 3 \mathrm{H}, \mathrm{CH}_{3}\right), 1.77-1.85(\mathrm{~m}, 2 \mathrm{H}$, $\left.\mathrm{CH}_{2}\right), 3.40\left(\mathrm{~s}, 3 \mathrm{H}, \mathrm{CH}_{3}\right), 3.66-3.78\left(\mathrm{~m}, 2 \mathrm{H}, \mathrm{CH}_{2}\right), 3.83-3.93\left(\mathrm{~m}, 2 \mathrm{H}, \mathrm{CH}_{2}\right), 7.96\left(\mathrm{t},{ }^{3} \mathrm{~J}(\mathrm{~F}, \mathrm{H})=58 \mathrm{~Hz}, 1 \mathrm{H}, \mathrm{CHF}_{2}\right) ;{ }^{13} \mathrm{C} \mathrm{NMR}^{2}$ $\left(100 \mathrm{MHz}, \mathrm{CDCl}_{3}, 25^{\circ} \mathrm{C}\right): \delta=9.0,13.3,19.5,24.4,43.7,55.4,58.8,113.7\left(\mathrm{t}, J=276 \mathrm{~Hz}, \mathrm{CHF}_{2}\right) ;{ }^{19} \mathrm{~F} \mathrm{NMR}\left(376 \mathrm{MHz} \mathrm{CDCl}_{3}\right.$, $\left.25^{\circ} \mathrm{C}\right): \delta=-117.36(\mathrm{~d}, J=59 \mathrm{~Hz}, 2 \mathrm{~F})$; Anal. calcd. for $\mathrm{C}_{8} \mathrm{H}_{18} \mathrm{~F}_{2} \mathrm{NI}: \mathrm{C}, 32.78, \mathrm{H}, 6.19, \mathrm{~N}, 4.78$. Found: $\mathrm{C}, 33.13, \mathrm{H}, 6.15, \mathrm{~N}$, 4.78 .

3i, $N$-difluoromethyl- $N, N, N$-triethylammonium iodide, m.p. $121-122^{\circ} \mathrm{C}$ decomp.; ${ }^{1} \mathrm{H}$ NMR $\left(400 \mathrm{MHz}, \mathrm{CDCl}_{3}, 25^{\circ} \mathrm{C}\right): \delta=$ $1.45\left(\mathrm{t},{ }^{3} J(\mathrm{H}, \mathrm{H})=7.4 \mathrm{~Hz}, 9 \mathrm{H}, 3 \mathrm{xCH}_{3}\right), 3.80\left(\mathrm{q},{ }^{3} J(\mathrm{H}, \mathrm{H})=7.3 \mathrm{~Hz}, 6 \mathrm{H}, 3 \mathrm{xCH}_{2}\right), 7.93\left(\mathrm{t},{ }^{3} J(\mathrm{~F}, \mathrm{H})=58 \mathrm{~Hz}, 1 \mathrm{H}, \mathrm{CHF}_{2}\right) ;{ }^{13} \mathrm{C} \mathrm{NMR}$ $\left(100 \mathrm{MHz}, \mathrm{CDCl}_{3}, 25^{\circ} \mathrm{C}\right): \delta=8.9,52.7,114.2\left(\mathrm{t}, J=275 \mathrm{~Hz}, \mathrm{CHF}_{2}\right) ;{ }^{19} \mathrm{~F}$ NMR $\left(376 \mathrm{MHz}, \mathrm{CDCl}_{3}, 25^{\circ} \mathrm{C}\right): \delta=-115.36(\mathrm{~d}, J=58$ $\mathrm{Hz}, 2 \mathrm{~F}$ ); Anal. calcd. for $\mathrm{C}_{7} \mathrm{H}_{16} \mathrm{~F}_{2} \mathrm{NI}$ : C, 30.12, H, 5.78, N, 5.02. Found: C, 30.38, H, 5.78, N, 5.04.

3j, $N$-difluoromethyl- $N$-methyl- $N$-piperidineammonium iodide, m.p. $115-117^{\circ} \mathrm{C}$ decomp.; ${ }^{1} \mathrm{H}$ NMR $\left(400 \mathrm{MHz}, \mathrm{CDCl}_{3}\right.$, $\left.25^{\circ} \mathrm{C}\right): \delta=1.64-1.75(\mathrm{~m}, 1 \mathrm{H}), 1.85-1.91(\mathrm{~m}, 1 \mathrm{H}), 1.98-2.13\left(\mathrm{~m}, 4 \mathrm{H}, 2 \mathrm{xCH}_{2}\right), 3.35\left(\mathrm{~s}, 3 \mathrm{H}_{1} \mathrm{CH}_{3}\right), 3.70-3.73\left(\mathrm{~m}, 2 \mathrm{H}, \mathrm{CH}_{2}\right), 3.91-$ $3.98\left(\mathrm{~m}, 2 \mathrm{H}, \mathrm{CH}_{2}\right), 7.99\left(\mathrm{t},{ }^{3} \mathrm{~J}(\mathrm{~F}, \mathrm{H})=59 \mathrm{~Hz}, 1 \mathrm{H}, \mathrm{CHF}_{2}\right) ;{ }^{13} \mathrm{C} \mathrm{NMR}\left(100 \mathrm{MHz}, \mathrm{CDCl}_{3}, 25^{\circ} \mathrm{C}\right): \delta=19.0,19.8,41.1,56.4,113.1$ $\left(\mathrm{t}, J=276 \mathrm{~Hz}, \mathrm{CHF}_{2}\right) ;{ }^{19} \mathrm{~F} \mathrm{NMR}\left(376 \mathrm{MHz}, \mathrm{CDCl}_{3}, 25^{\circ} \mathrm{C}\right): \delta=-121.81(\mathrm{~d}, J=60 \mathrm{~Hz}, 2 \mathrm{~F})$; Anal. calcd. for $\mathrm{C}_{7} \mathrm{H}_{14} \mathrm{~F}_{2} \mathrm{NI}: \mathrm{C}_{\text {, }}$ $30.34, \mathrm{H}, 5.09, \mathrm{~N}, 5.05$. Found: C, 30.54, H, 5.11, N, 5.10.

3k, $N$-cyclohexyl- $N$-difluoromethyl- $N, N$-dimethylammonium iodide, m.p. $110-111^{\circ} \mathrm{C}$ decomp.; ${ }^{1} \mathrm{H}$ NMR $(400 \mathrm{MHz}$, $\left.\mathrm{CDCl}_{3}, 25^{\circ} \mathrm{C}\right): \delta=1.06-1.18(\mathrm{~m}, 1 \mathrm{H}), 1.34-1.45\left(\mathrm{~m}, 2 \mathrm{H}, \mathrm{CH}_{2}\right), 1.55-1.64(\mathrm{~m}, 3 \mathrm{H}), 1.90-1.93\left(\mathrm{~m}, 2 \mathrm{H}, \mathrm{CH}_{2}\right), 2.29-2.32(\mathrm{~m}, 2 \mathrm{H}$, $\left.\mathrm{CH}_{2}\right), 3.35\left(\mathrm{~s}, 6 \mathrm{H}, 2 \mathrm{xCH}_{3}\right), 3.97-4.05(\mathrm{~m}, 1 \mathrm{H}, \mathrm{CH}), 7.89\left(\mathrm{t},{ }^{3} \mathrm{~J}(\mathrm{~F}, \mathrm{H})=58 \mathrm{~Hz}, 1 \mathrm{H}, \mathrm{CHF}_{2}\right) ;{ }^{13} \mathrm{C} \mathrm{NMR}\left(100 \mathrm{MHz}, \mathrm{CDCl}_{3}, 25^{\circ} \mathrm{C}\right)$ : $\delta=24.1,26.0,27.2,44.0,74.7,113.2\left(\mathrm{t}, J=275 \mathrm{~Hz}, \mathrm{CHF}_{2}\right) ;{ }^{19} \mathrm{~F}$ NMR $\left(376 \mathrm{MHz}, \mathrm{CDCl}_{3}, 25^{\circ} \mathrm{C}\right): \delta=-117.87(\mathrm{~d}, J=58 \mathrm{~Hz}$, 2F); Anal. calcd. for $\mathrm{C}_{9} \mathrm{H}_{18} \mathrm{~F}_{2} \mathrm{NI}$ : C, 35.43, H, 5.95, N, 4.59. Found: C, 35.70, H, 6.08, N, 4.77.

31, $N$-difluoromethyl- $N, N$-dimethyl- $N$-piridin-4-ammonium iodide, m.p. $143-145^{\circ} \mathrm{C} ;{ }^{1} \mathrm{H}$ NMR $\left(400 \mathrm{MHz}, \mathrm{DMSO}, 25^{\circ} \mathrm{C}\right)$ : $\delta=3.30\left(\mathrm{~s}, 6 \mathrm{H}, 2 \mathrm{xCH}_{3}\right), 7.17-7.21(\mathrm{~m}, 2 \mathrm{H}, \mathrm{ArH}), 7.83\left(\mathrm{t},{ }^{3} J(\mathrm{~F}, \mathrm{H})=59 \mathrm{~Hz}, 1 \mathrm{H}, \mathrm{CHF}_{2}\right), 8.53-8.56(\mathrm{~m}, 2 \mathrm{H}, \mathrm{ArH}) ;{ }^{13} \mathrm{C} \mathrm{NMR}(100$ MHz, DMSO, $\left.25^{\circ} \mathrm{C}\right): \delta=40.7,107.9,111.9\left(\mathrm{t}, J=255 \mathrm{~Hz}, \mathrm{CHF}_{2}\right), 137.0,157.4 ;{ }^{19} \mathrm{~F}$ NMR $\left(376 \mathrm{MHz}\right.$, DMSO, $\left.25^{\circ} \mathrm{C}\right): \delta=-$ $94.73(\mathrm{~d}, J=58 \mathrm{~Hz}, 2 \mathrm{~F})$; Anal. calcd. for $\mathrm{C}_{8} \mathrm{H}_{11} \mathrm{~F}_{2} \mathrm{~N}_{2} \mathrm{I}: \mathrm{C}, 32.02, \mathrm{H}, 3.69, \mathrm{~N}, 9.33$. Found: C, 32.20, H, 3.77, N, 9.26.

4h, $N$-buthyl- $N$-difluoromethyl- $N$-ethyl- $N$-methylammonium nitrate, m.p. $52-54^{\circ} \mathrm{C}$; ${ }^{1} \mathrm{H}$ NMR $\left(400 \mathrm{MHz}, \mathrm{CDCl}_{3}, 25^{\circ} \mathrm{C}\right)$ : $\delta=0.91\left(\mathrm{t},{ }^{3} J(\mathrm{H}, \mathrm{H})=7.4 \mathrm{~Hz}, 3 \mathrm{H}, \mathrm{CH}_{3}\right), 1.30-1.39\left(\mathrm{~m}, 2 \mathrm{H}, \mathrm{CH}_{2}\right), 1.43\left(\mathrm{t},{ }^{3} J(\mathrm{H}, \mathrm{H})=7.2 \mathrm{~Hz}, 3 \mathrm{H}, \mathrm{CH}_{3}\right), 1.72-1.80\left(\mathrm{~m}, 2 \mathrm{H}, \mathrm{CH}_{2}\right)$, $3.26\left(\mathrm{~s}, 3 \mathrm{H}, \mathrm{CH}_{3}\right), 3.54-3.60\left(\mathrm{~m}, 2 \mathrm{H}, \mathrm{CH}_{2}\right), 3.69-3.76\left(\mathrm{~m}, 2 \mathrm{H}, \mathrm{CH}_{2}\right), 7.71\left(\mathrm{t},{ }^{3} \mathrm{~J}(\mathrm{~F}, \mathrm{H})=58 \mathrm{~Hz}, 1 \mathrm{H}, \mathrm{CHF}_{2}\right) ;{ }^{13} \mathrm{C} \mathrm{NMR}(100 \mathrm{MHz}$, $\left.\mathrm{CDCl}_{3}, 25^{\circ} \mathrm{C}\right): \delta=8.1,13.2,19.6,24.0,42.6,54.8,58.4,115.1\left(\mathrm{t}, J=274 \mathrm{~Hz}, \mathrm{CHF}_{2}\right) ;{ }^{19} \mathrm{~F} \mathrm{NMR}\left(376 \mathrm{MHz}, \mathrm{CDCl}_{3}, 25^{\circ} \mathrm{C}\right): \delta=-$ 117.92 (d, $J=58 \mathrm{~Hz}, 2 \mathrm{~F})$; Anal. calcd. for $\mathrm{C}_{8} \mathrm{H}_{18} \mathrm{~F}_{2} \mathrm{~N}_{2} \mathrm{O}_{3}: \mathrm{C}, 42.10, \mathrm{H}, 7.95, \mathrm{~N}, 12.27$. Found: $\mathrm{C}, 42.08, \mathrm{H}, 7.72, \mathrm{~N}, 11.90$.

4i, $N$-difluoromethyl- $N, N, N$-triethylammonium nitrate, m.p. $142-145^{\circ} \mathrm{C}$ decomp.; ${ }^{1} \mathrm{H}$ NMR $\left(400 \mathrm{MHz}, \mathrm{CDCl}_{3}, 25^{\circ} \mathrm{C}\right): \delta=$ $1.41\left(\mathrm{t},{ }^{3} J(\mathrm{H}, \mathrm{H})=7.2 \mathrm{~Hz}, 9 \mathrm{H}, 3 \mathrm{xCH}_{3}\right), 3.70\left(\mathrm{q},{ }^{3} \mathrm{~J}(\mathrm{H}, \mathrm{H})=7.3 \mathrm{~Hz}, 6 \mathrm{H}, 3 \mathrm{xCH}_{2}\right), 7.74\left(\mathrm{t},{ }^{3} \mathrm{~J}(\mathrm{~F}, \mathrm{H})=57 \mathrm{~Hz}, 1 \mathrm{H}, \mathrm{CHF}_{2}\right) ;{ }^{13} \mathrm{C}_{\mathrm{NMR}}$ $\left(100 \mathrm{MHz}, \mathrm{CDCl}_{3}, 25^{\circ} \mathrm{C}\right): \delta=8.0,52.1,115.5\left(\mathrm{t}, J=274 \mathrm{~Hz}, \mathrm{CHF}_{2}\right) ;{ }^{19} \mathrm{~F}$ NMR $\left(376 \mathrm{MHz}, \mathrm{CDCl}_{3}, 25^{\circ} \mathrm{C}\right): \delta=-115.82(\mathrm{~d}, J=56$ $\mathrm{Hz}, 2 \mathrm{~F}$ ); Anal. calcd. for $\mathrm{C}_{7} \mathrm{H}_{16} \mathrm{~F}_{2} \mathrm{~N}_{2} \mathrm{O}_{3}$ : C, 39.25, H, 7.53, N, 13.08. Found: C, 39.48, H, 7.38, N, 12.96 .

4j, $N$-difluoromethyl- $N$-methyl- $N$-piperidineammonium nitrate, m.p. $165-166{ }^{\circ} \mathrm{C} ;{ }^{1} \mathrm{H}$ NMR $\left(400 \mathrm{MHz}, \mathrm{DMSO}, 25^{\circ} \mathrm{C}\right)$ : $\delta=1.54-1.59(\mathrm{~m}, 1 \mathrm{H}), 1.79-1.84(\mathrm{~m}, 1 \mathrm{H}), 1.91-2.00\left(\mathrm{~m}, 4 \mathrm{H}, 2 \mathrm{xCH}_{2}\right), 3.25\left(\mathrm{~s}, 3 \mathrm{H}, \mathrm{CH}_{3}\right), 3.55-3.58\left(\mathrm{~m}, 2 \mathrm{H}, \mathrm{CH}_{2}\right), 3.69-3.75$ $\left(\mathrm{m}, 2 \mathrm{H}, \mathrm{CH}_{2}\right), 7.69\left(\mathrm{t},{ }^{3} J(\mathrm{~F}, \mathrm{H})=59 \mathrm{~Hz}, 1 \mathrm{H}, \mathrm{CHF}_{2}\right) ;{ }^{13} \mathrm{C}$ NMR $\left(100 \mathrm{MHz}, \mathrm{DMSO}, 25^{\circ} \mathrm{C}\right): \delta=18.9,20.4,39.9,56.3,115.1(\mathrm{t}, J=$ $\left.274 \mathrm{~Hz}, \mathrm{CHF}_{2}\right) ;{ }^{19} \mathrm{~F}$ NMR $\left(376 \mathrm{MHz}, \mathrm{DMSO}, 25^{\circ} \mathrm{C}\right): \delta=-121.94(\mathrm{~d}, J=59 \mathrm{~Hz}, 2 \mathrm{~F}) ; \mathrm{HRMS}$ for $\left[\mathrm{C}_{7} \mathrm{H}_{14} \mathrm{NF}_{2}\right]^{+}: 150.10888$. Found: 150.10863

4k, $N$-cyclohexyl- $N$-difluoromethyl- $N, N$-dimethylammonium nitrate, m.p. $115-118^{\circ} \mathrm{C}$ decomp.; ${ }^{1} \mathrm{H}$ NMR $(400 \mathrm{MHz}$, $\left.\mathrm{CDCl}_{3}, 25^{\circ} \mathrm{C}\right): \delta=1.05-1.17(\mathrm{~m}, 1 \mathrm{H}), 1.29-1.40\left(\mathrm{~m}, 2 \mathrm{H}, \mathrm{CH}_{2}\right), 1.52-1.64(\mathrm{~m}, 3 \mathrm{H}), 1.90-1.94\left(\mathrm{~m}, 2 \mathrm{H}, \mathrm{CH}_{2}\right), 2.26-2.28(\mathrm{~m}, 2 \mathrm{H}$, $\left.\mathrm{CH}_{2}\right), 3.24\left(\mathrm{~s}, 6 \mathrm{H}, 2 \mathrm{xCH}_{3}\right), 3.73-3.80(\mathrm{~m}, 1 \mathrm{H}, \mathrm{CH}), 7.71\left(\mathrm{t},{ }^{3} \mathrm{~J}(\mathrm{~F}, \mathrm{H})=58 \mathrm{~Hz}, 1 \mathrm{H}, \mathrm{CHF}_{2}\right) ;{ }^{13} \mathrm{C} \mathrm{NMR}\left(100 \mathrm{MHz}, \mathrm{CDCl}_{3}, 25^{\circ} \mathrm{C}\right)$ : $\delta=24.4,25.3,26.6,42.8,74.6,114.6\left(\mathrm{t}, J=274 \mathrm{~Hz}, \mathrm{CHF}_{2}\right) ;{ }^{19} \mathrm{~F}$ NMR $\left(376 \mathrm{MHz}, \mathrm{CDCl}_{3}, 25^{\circ} \mathrm{C}\right): \delta=-118.35(\mathrm{~d}, J=58 \mathrm{~Hz}$, 2F); Anal. calcd. for $\mathrm{C}_{9} \mathrm{H}_{18} \mathrm{~F}_{2} \mathrm{~N}_{2} \mathrm{O}_{3}$ : C, 44.99, $\mathrm{H}, 7.55, \mathrm{~N}, 11.66$. Found: C, 45.27, H, 7.43, N, 11.44. 
5i, $N$-difluoromethyl- $N, N, N$-triethylammonium thiocyanate, m.p. $46-48^{\circ} \mathrm{C} ;{ }^{1} \mathrm{H}$ NMR $\left(400 \mathrm{MHz}, \mathrm{CDCl}_{3}, 25^{\circ} \mathrm{C}\right): \delta=1.57$ (t, $\left.{ }^{3} J(\mathrm{H}, \mathrm{H})=7.4 \mathrm{~Hz}, 9 \mathrm{H}, 3 \mathrm{xCH}_{3}\right), 3.81\left(\mathrm{q},{ }^{3} J(\mathrm{H}, \mathrm{H})=7.2 \mathrm{~Hz}, 6 \mathrm{H}, 3 \mathrm{xCH}_{2}\right), 7.58\left(\mathrm{t},{ }^{3} J(\mathrm{~F}, \mathrm{H})=58 \mathrm{~Hz}, 1 \mathrm{H}, \mathrm{CHF}_{2}\right) ;{ }^{13} \mathrm{C} \mathrm{NMR}(100$ $\left.\mathrm{MHz}, \mathrm{CDCl}_{3}, 25^{\circ} \mathrm{C}\right): \delta=8.6,52.7,115.3\left(\mathrm{t}, J=275 \mathrm{~Hz}, \mathrm{CHF}_{2}\right), 131.7(\mathrm{SCN}) ;{ }^{19} \mathrm{~F} \mathrm{NMR}\left(376 \mathrm{MHz}, \mathrm{CDCl}_{3}, 25^{\circ} \mathrm{C}\right): \delta=-114.79$ $(\mathrm{d}, J=58 \mathrm{~Hz}, 2 \mathrm{~F})$; HRMS for $\left[\mathrm{C}_{7} \mathrm{H}_{16} \mathrm{NF}_{2}\right]^{+}: 152.12453$. Found: 152.12519.

5j, $N$-difluoromethyl- $N$-methyl- $N$-piperidineammonium thiocyanate, ${ }^{1} \mathrm{H}$ NMR $\left(400 \mathrm{MHz},\left(\mathrm{CD}_{3}\right)_{2} \mathrm{CO}, 25^{\circ} \mathrm{C}\right): \delta=1.73-1.79$ $(\mathrm{m}, 1 \mathrm{H}), 1.86-1.90(\mathrm{~m}, 1 \mathrm{H}), 2.06-2.08\left(\mathrm{~m}, 2 \mathrm{H}, \mathrm{CH}_{2}\right), 2.16-2.22\left(\mathrm{~m}, 2 \mathrm{H}, \mathrm{CH}_{2}\right), 3.47\left(\mathrm{~s}, 3 \mathrm{H}, \mathrm{CH}_{3}\right), 3.78-3.90\left(\mathrm{~m}, 4 \mathrm{H}, \mathrm{CH}_{2}\right)$, $7.59\left(\mathrm{t},{ }^{3} J(\mathrm{~F}, \mathrm{H})=59 \mathrm{~Hz}, 1 \mathrm{H}, \mathrm{CHF}_{2}\right) ;{ }^{13} \mathrm{C} \mathrm{NMR}\left(100 \mathrm{MHz},\left(\mathrm{CD}_{3}\right)_{2} \mathrm{CO}, 25^{\circ} \mathrm{C}\right): \delta=19.6,21.2,40.7,57.4,115.8(\mathrm{t}, J=272 \mathrm{~Hz}$, $\left.\mathrm{CHF}_{2}\right), 132.3(\mathrm{SCN}) ;{ }^{19} \mathrm{~F}$ NMR $\left(376 \mathrm{MHz},\left(\mathrm{CD}_{3}\right)_{2} \mathrm{CO}, 25^{\circ} \mathrm{C}\right): \delta=-117.80(\mathrm{~d}, J=59 \mathrm{~Hz}, 2 \mathrm{~F})$; HRMS for $\left[\mathrm{C}_{7} \mathrm{H}_{14} \mathrm{NF}_{2}\right]^{+}$: 150.10888. Found: 150.10929 . 


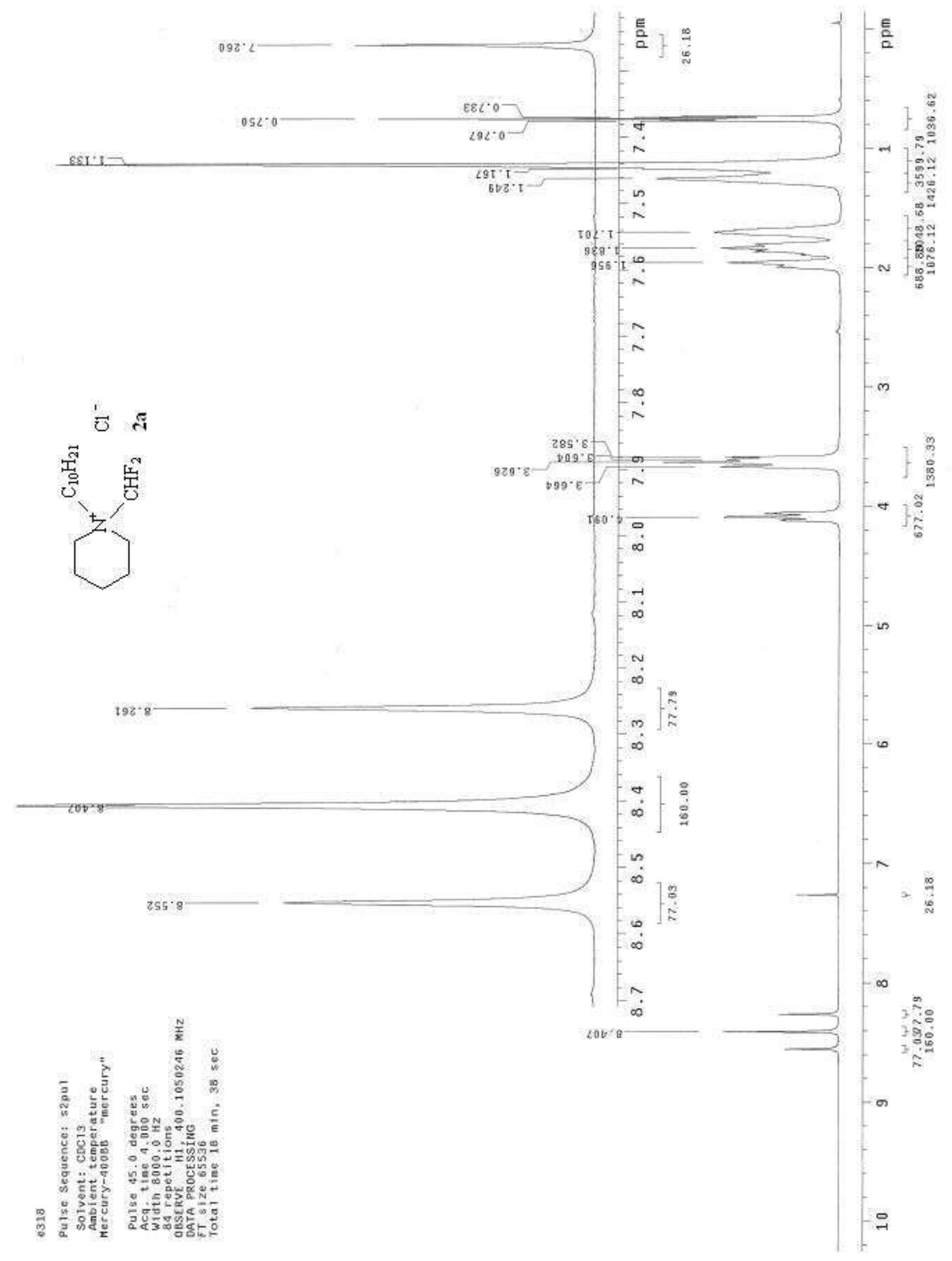




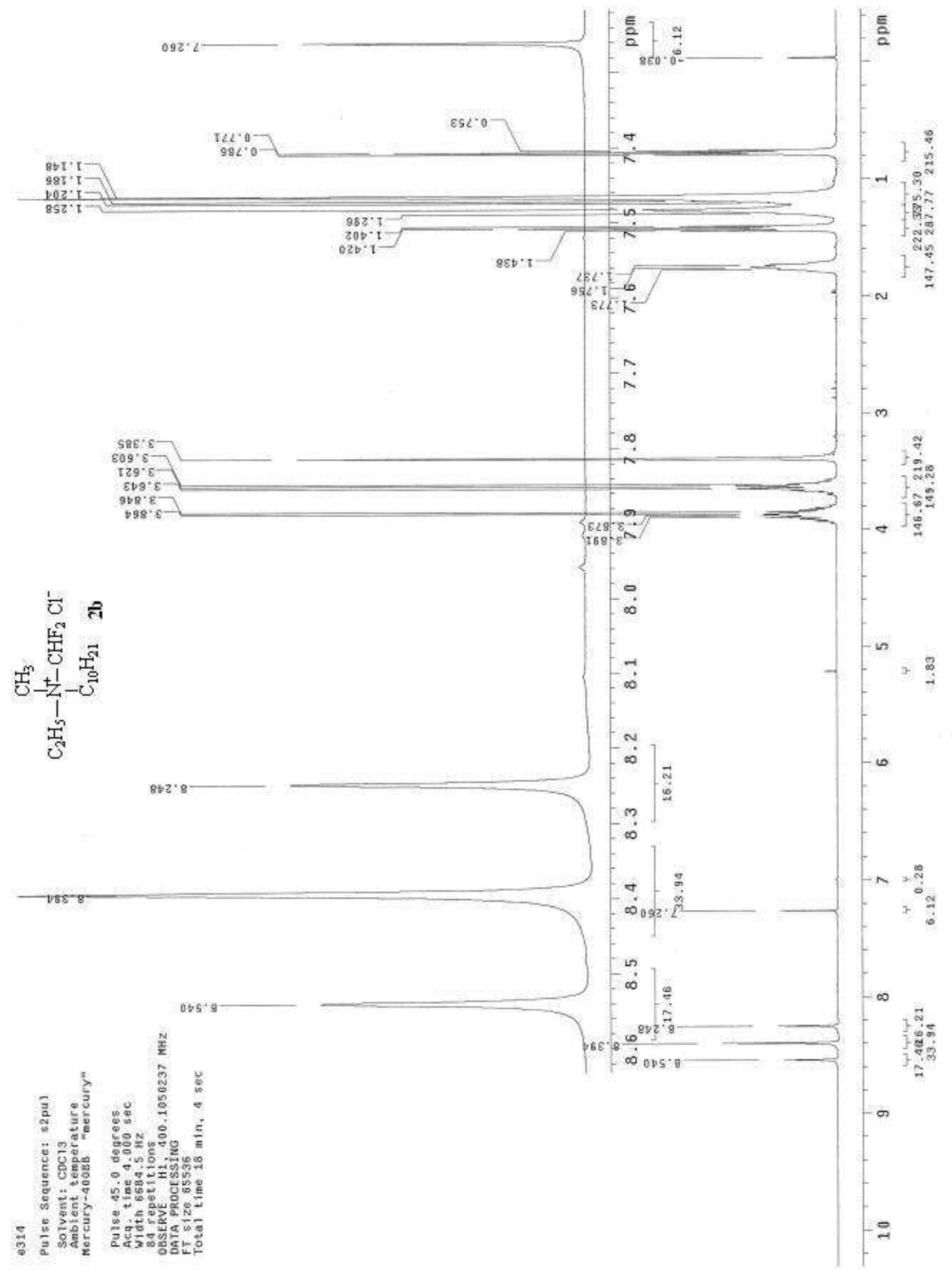




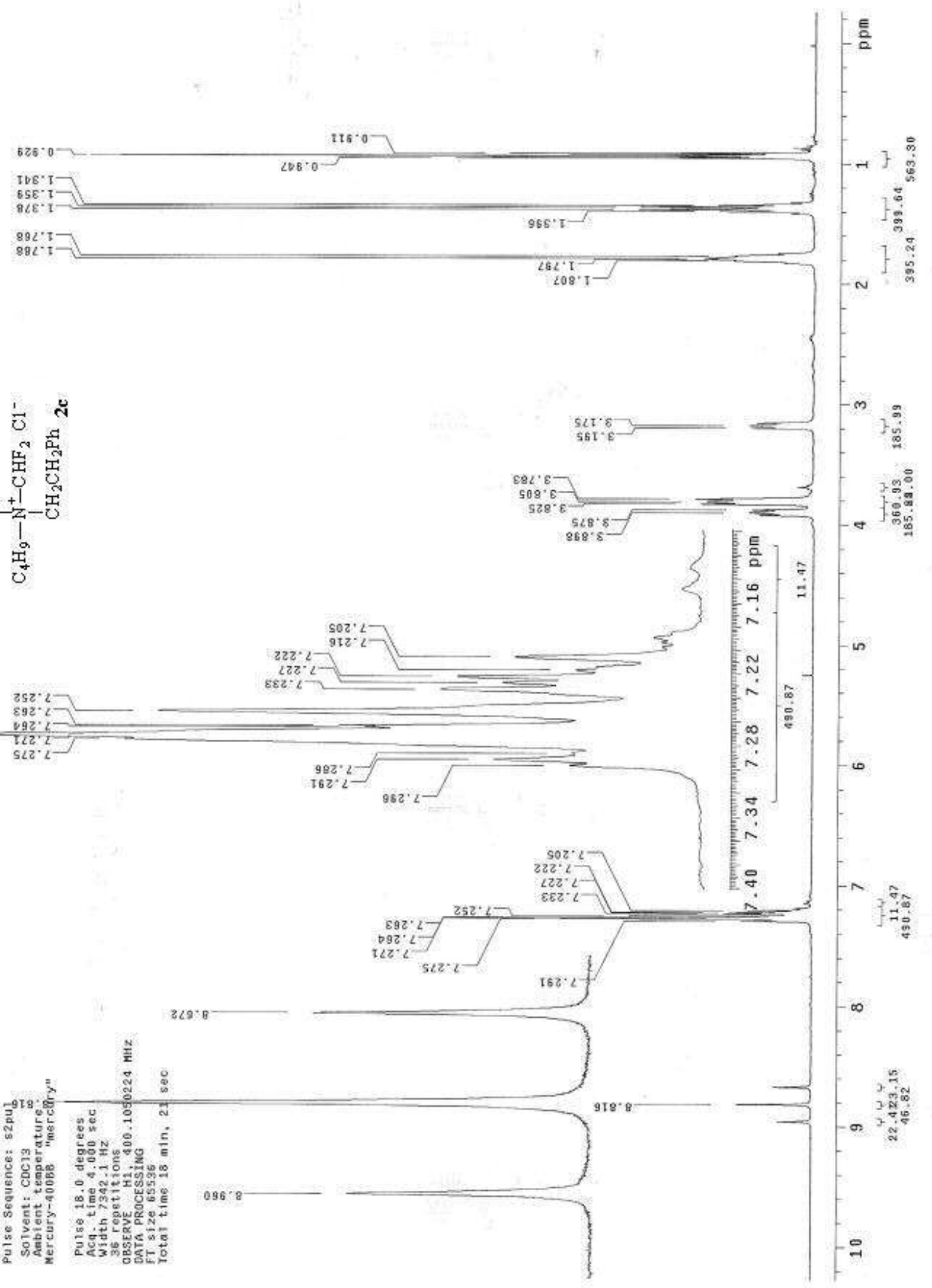




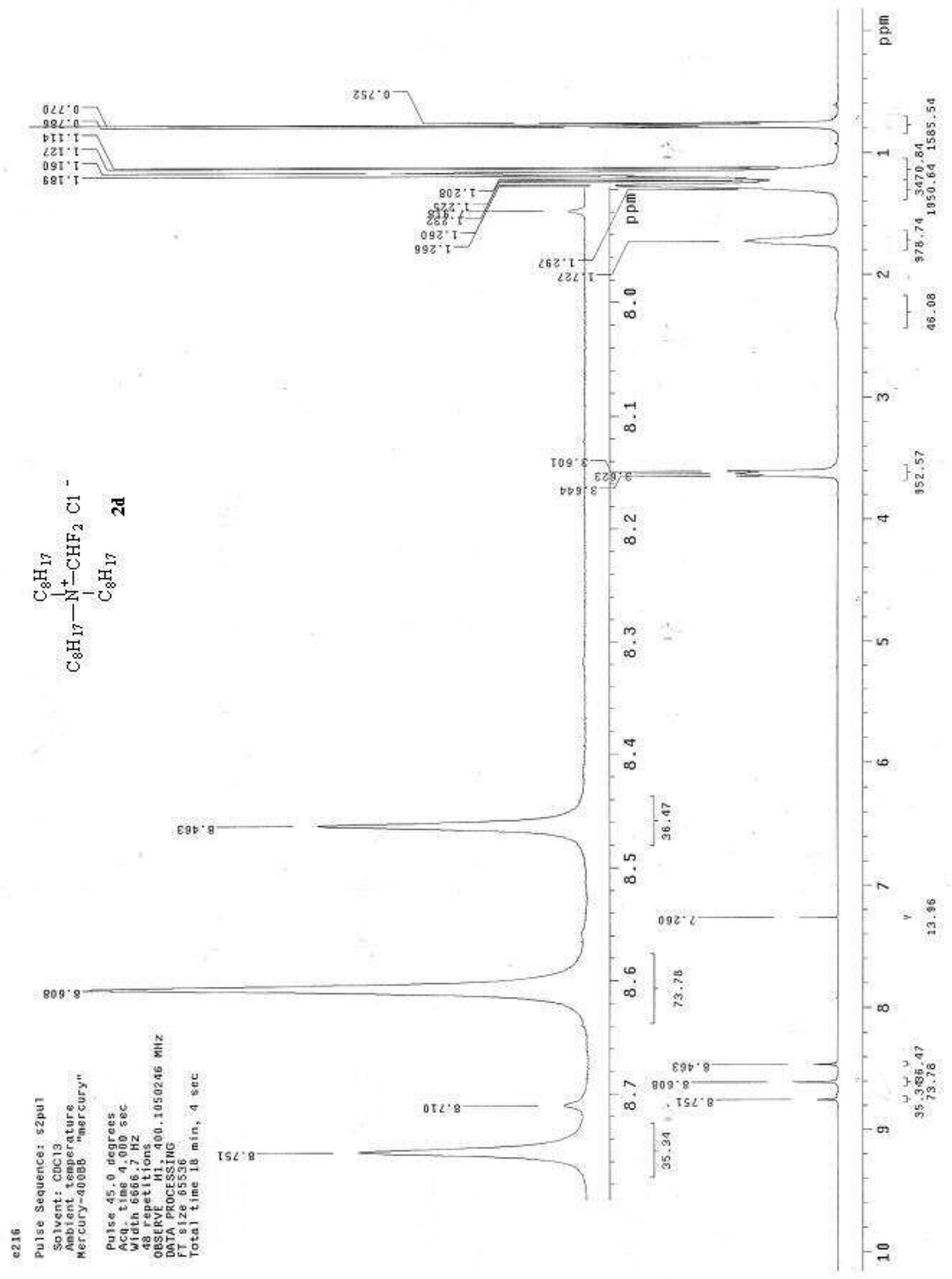




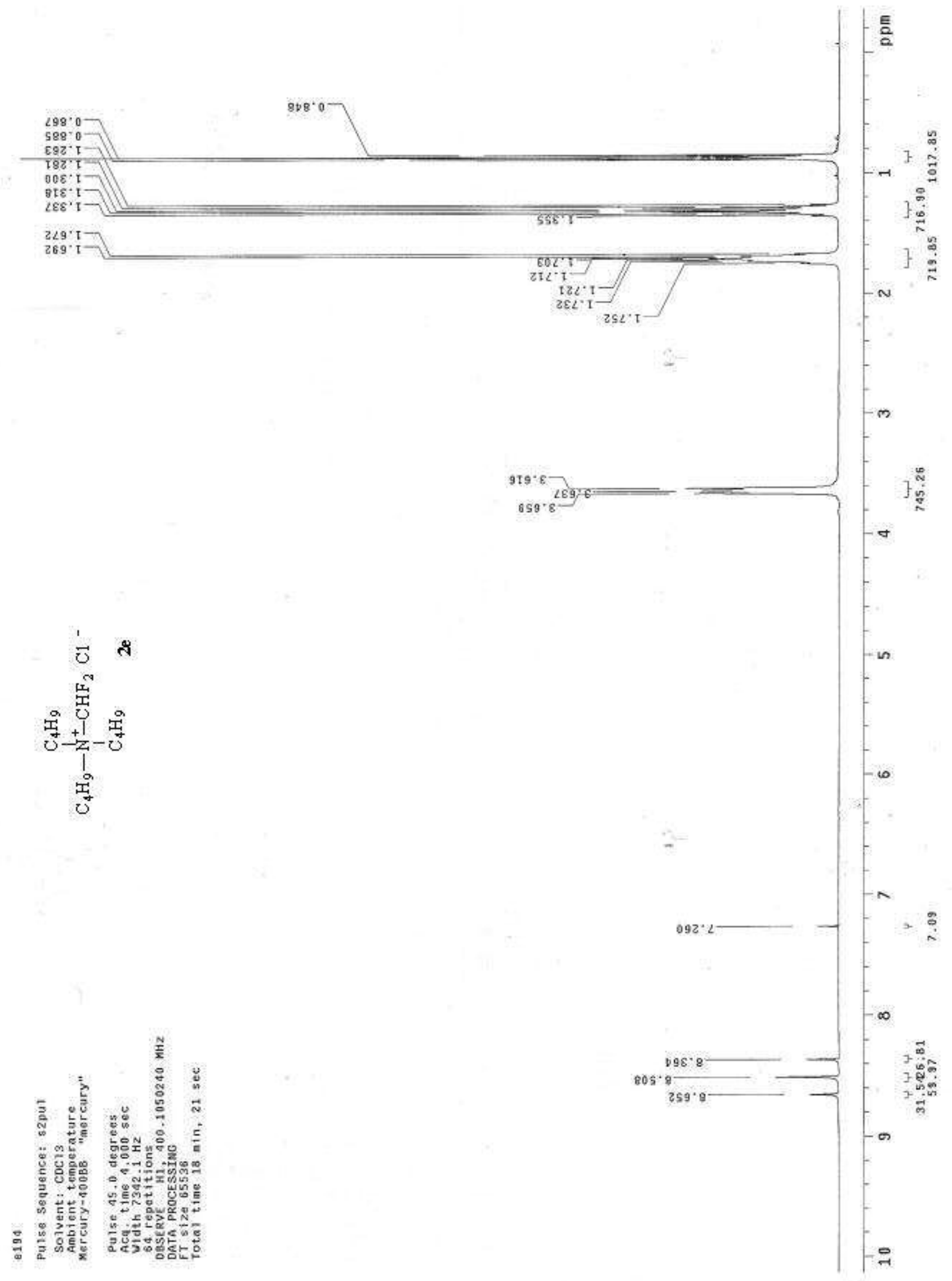




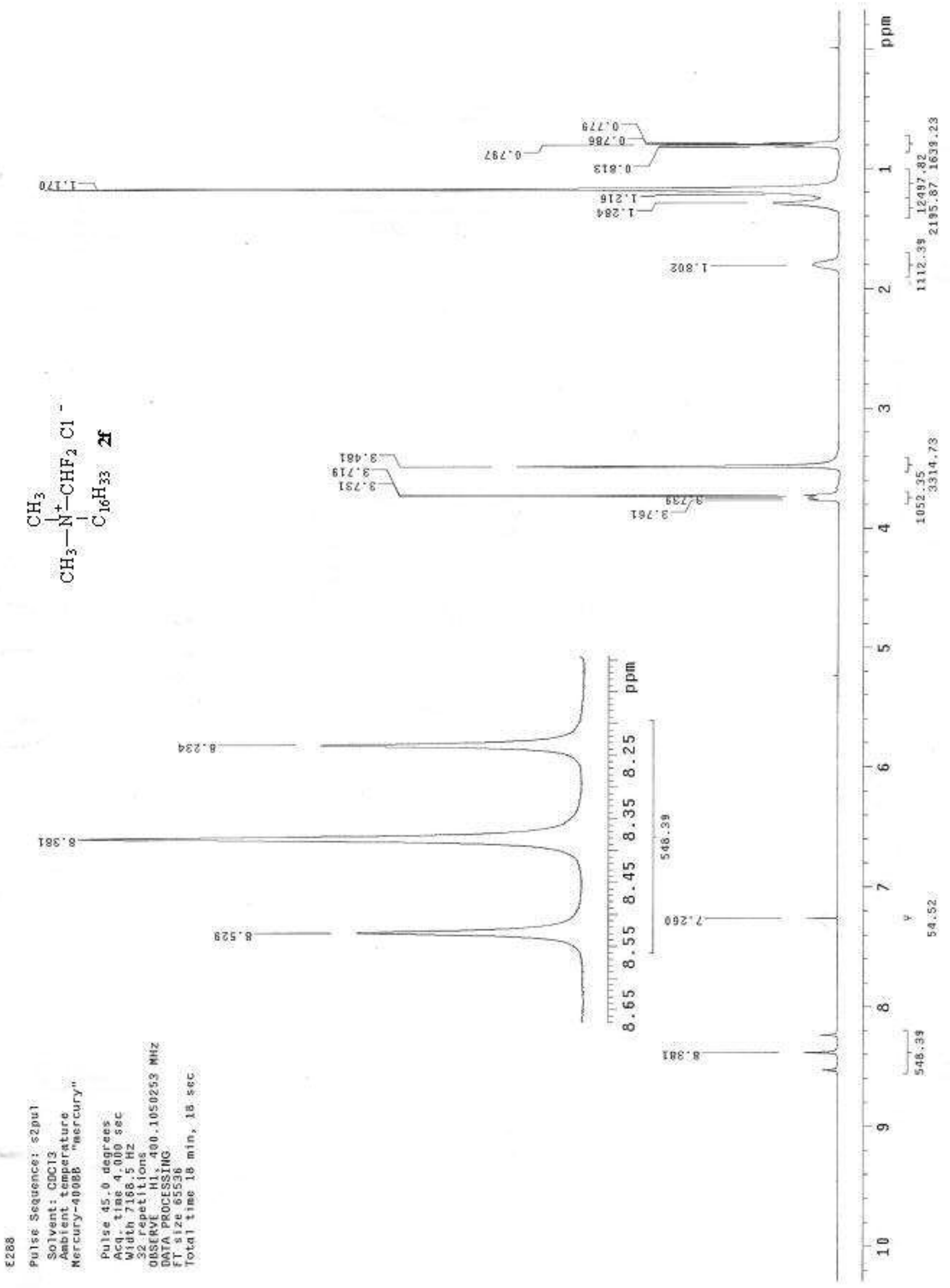




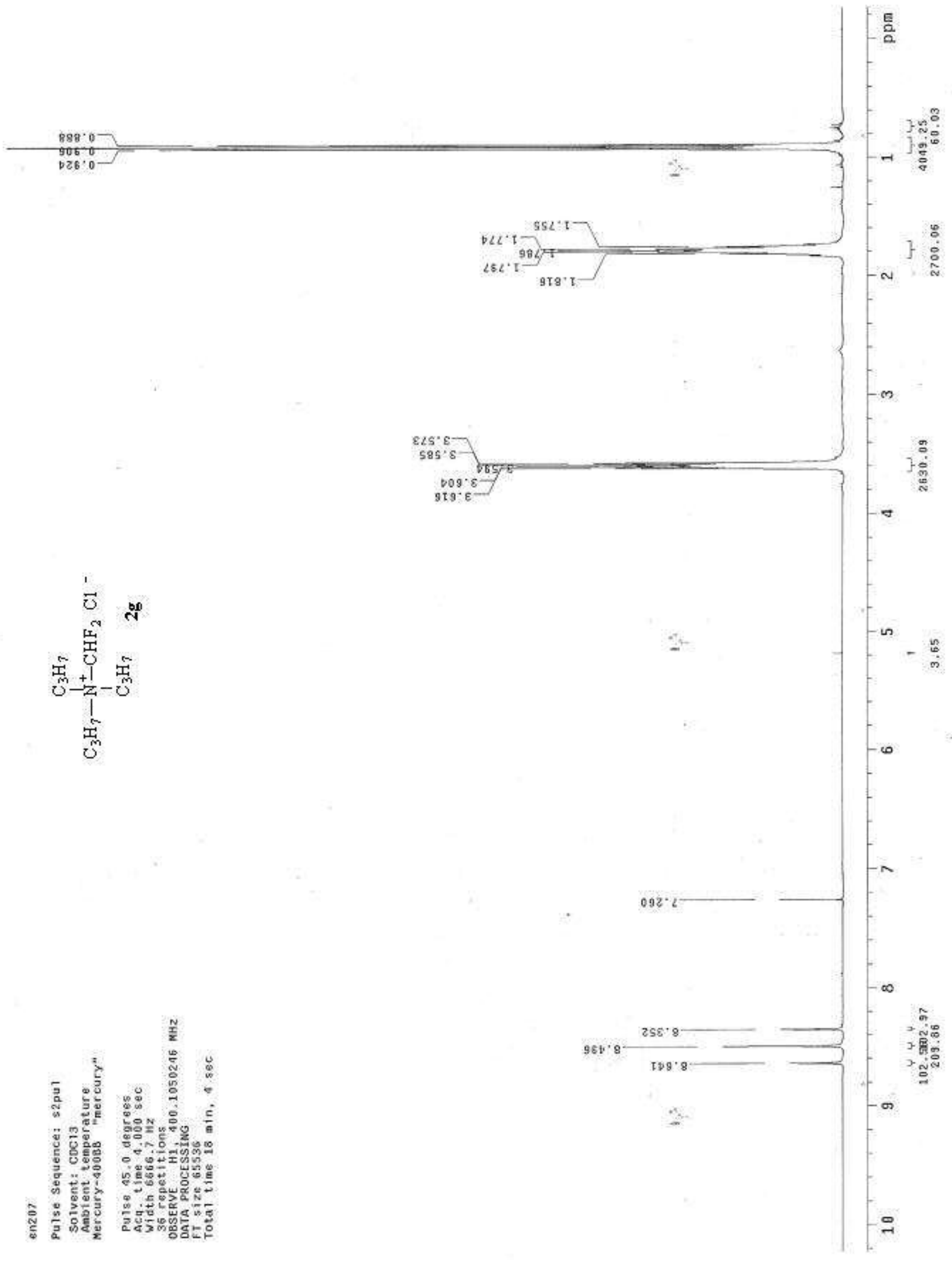




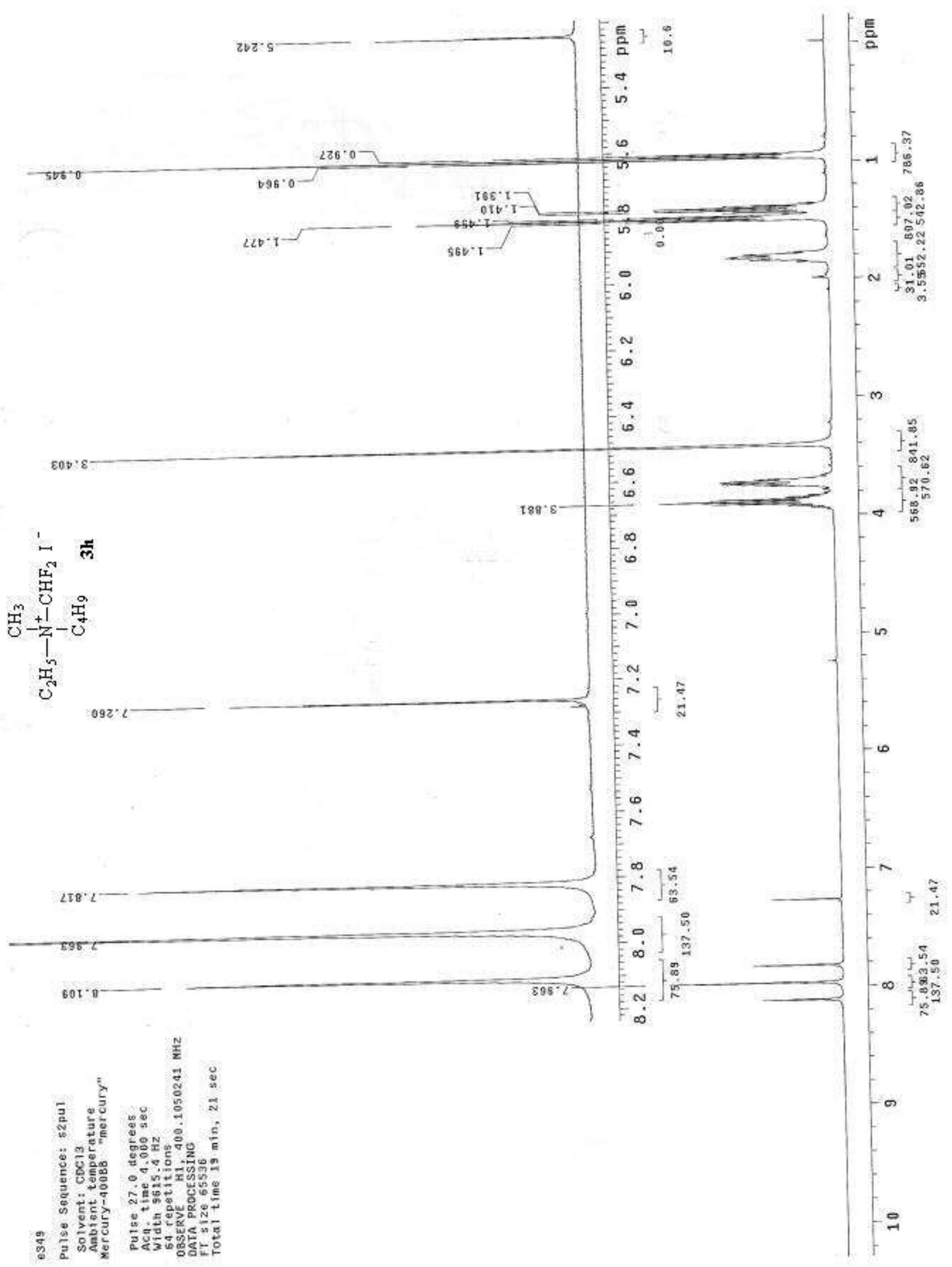




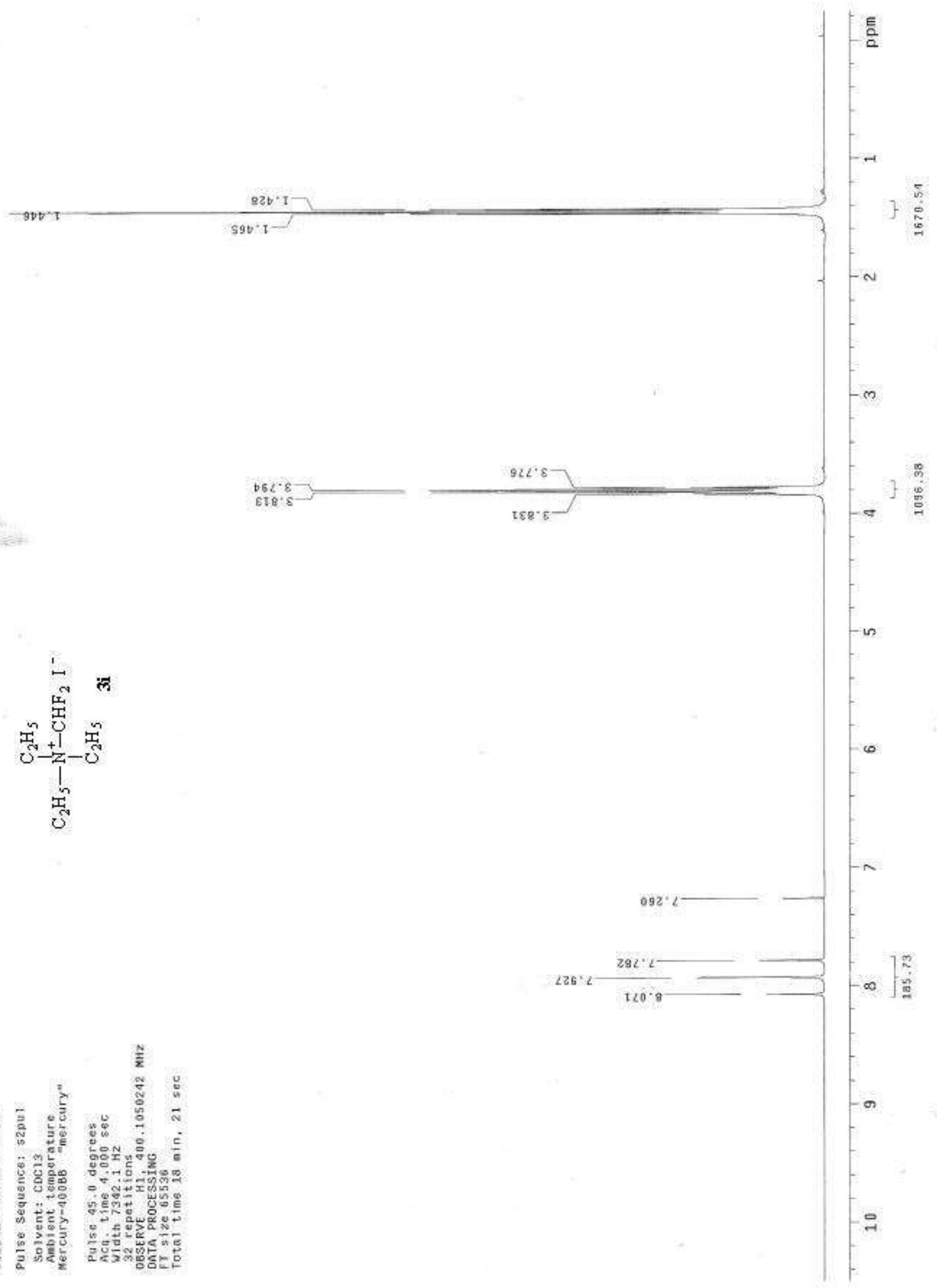




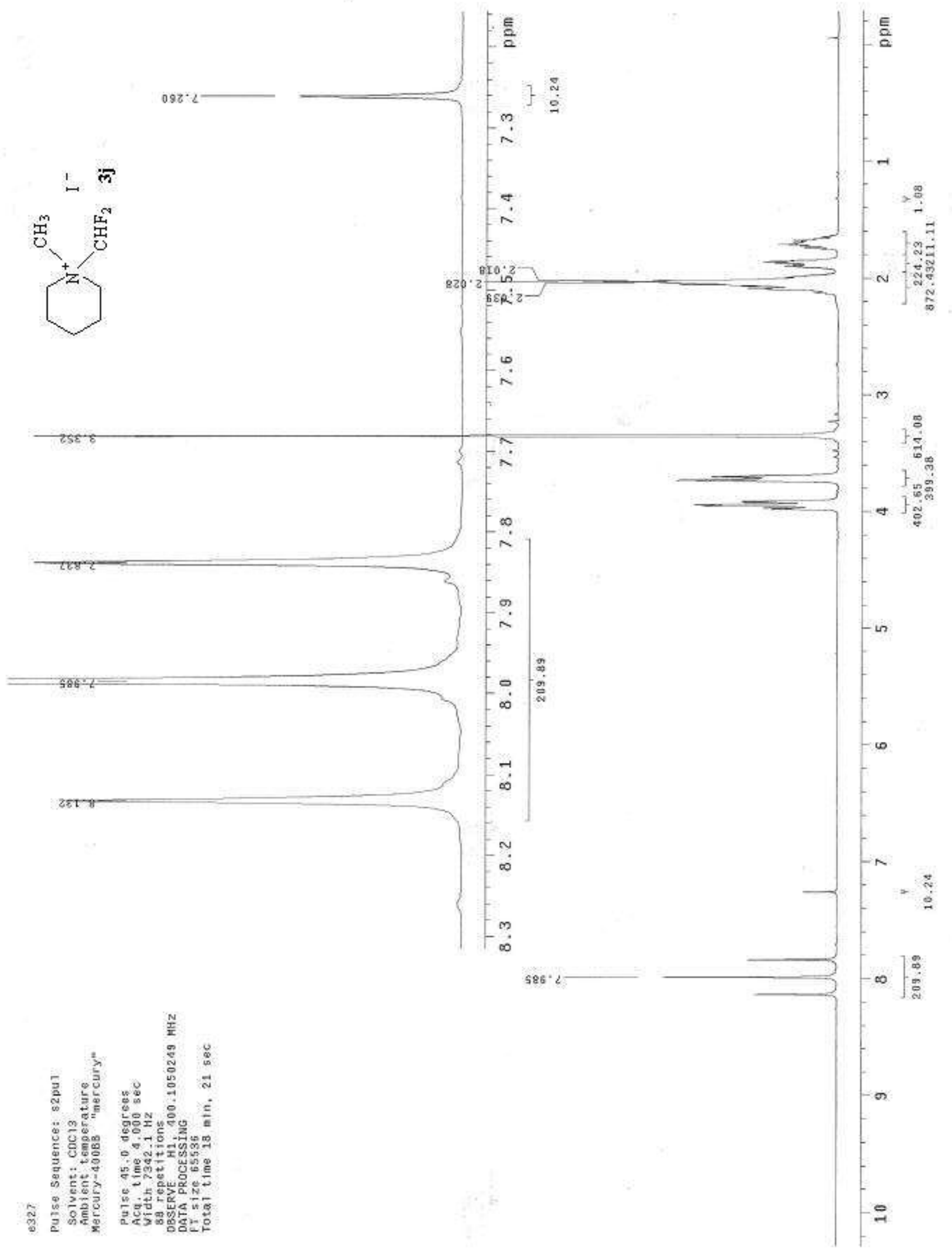




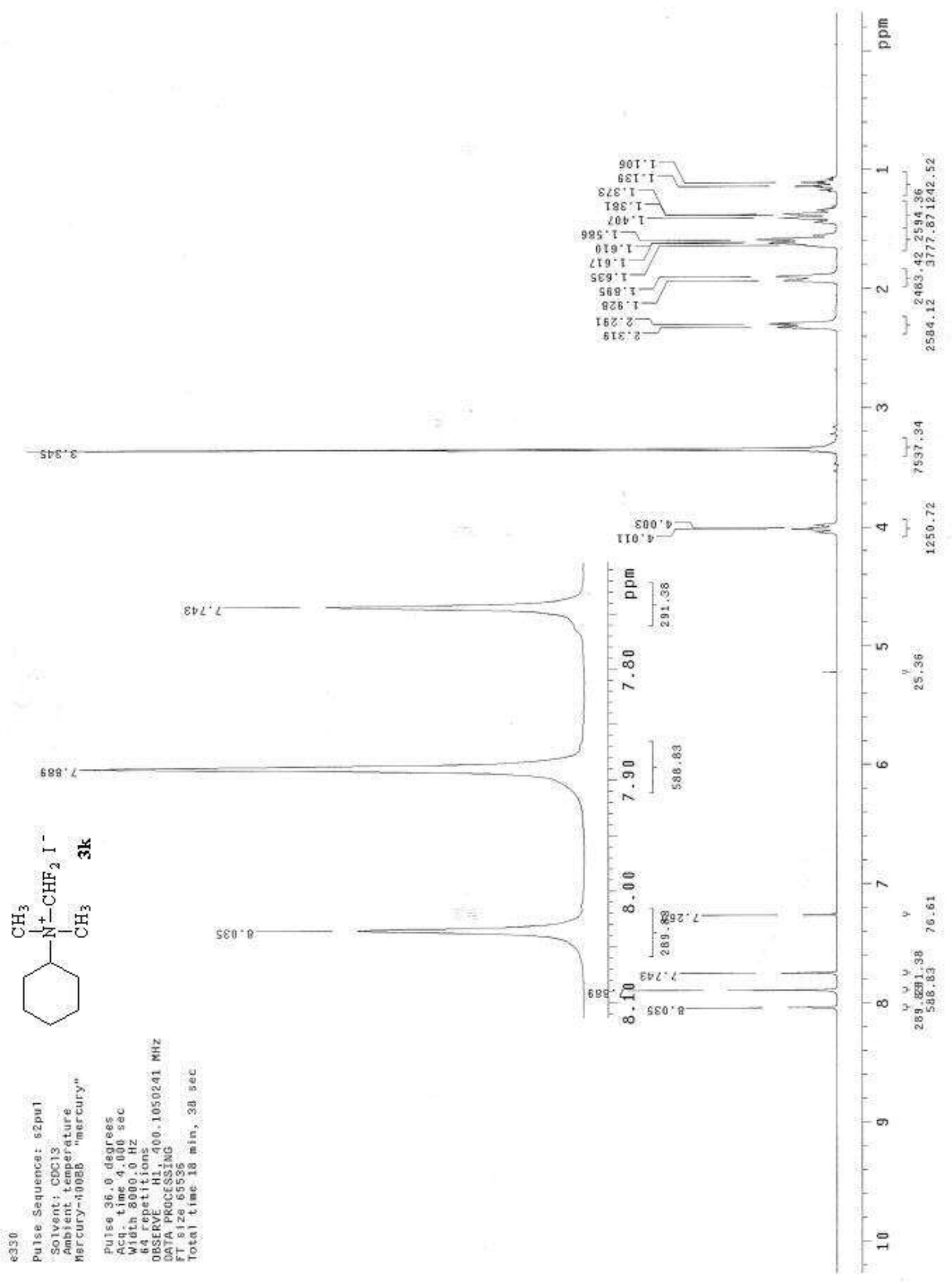




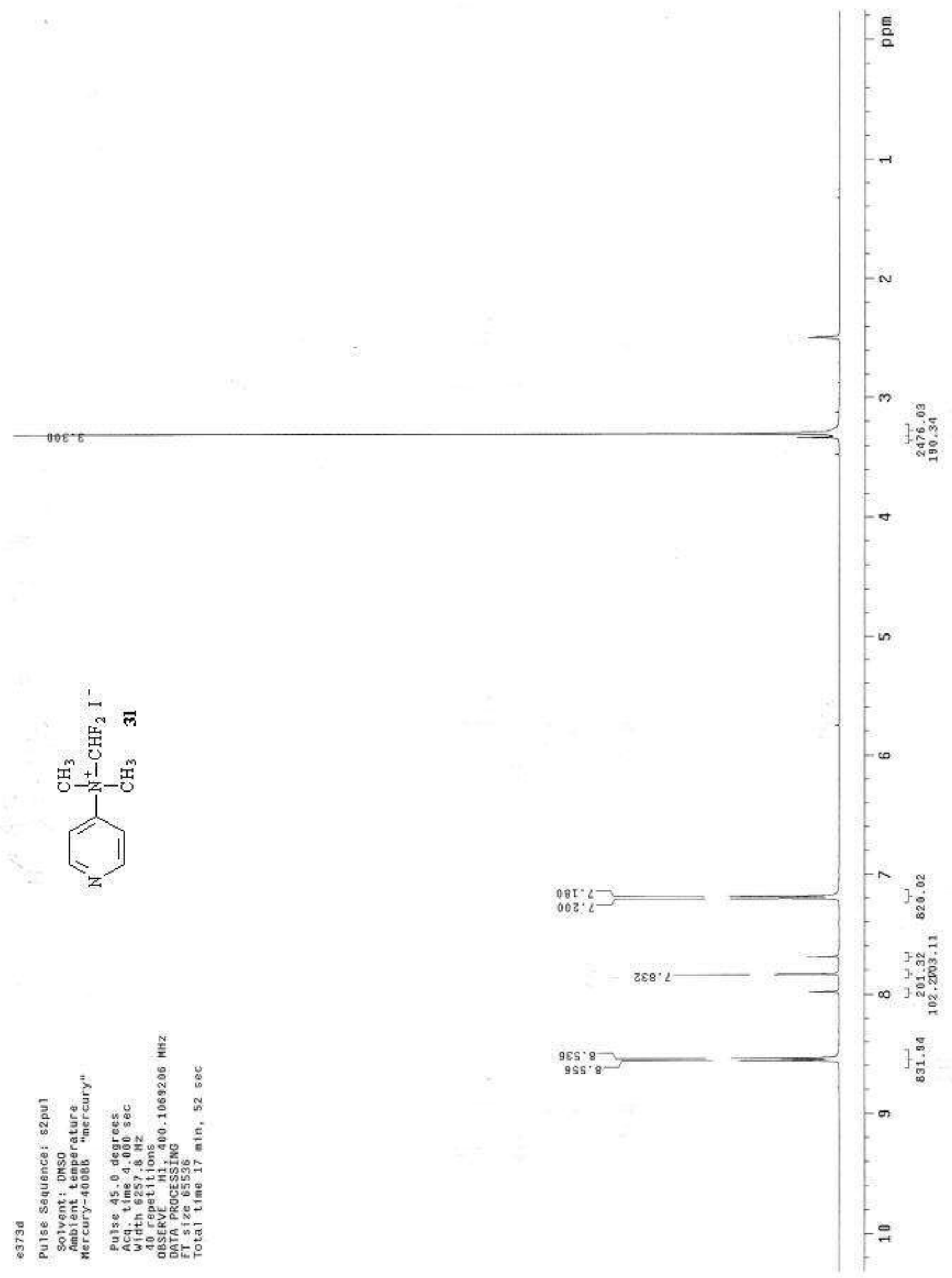




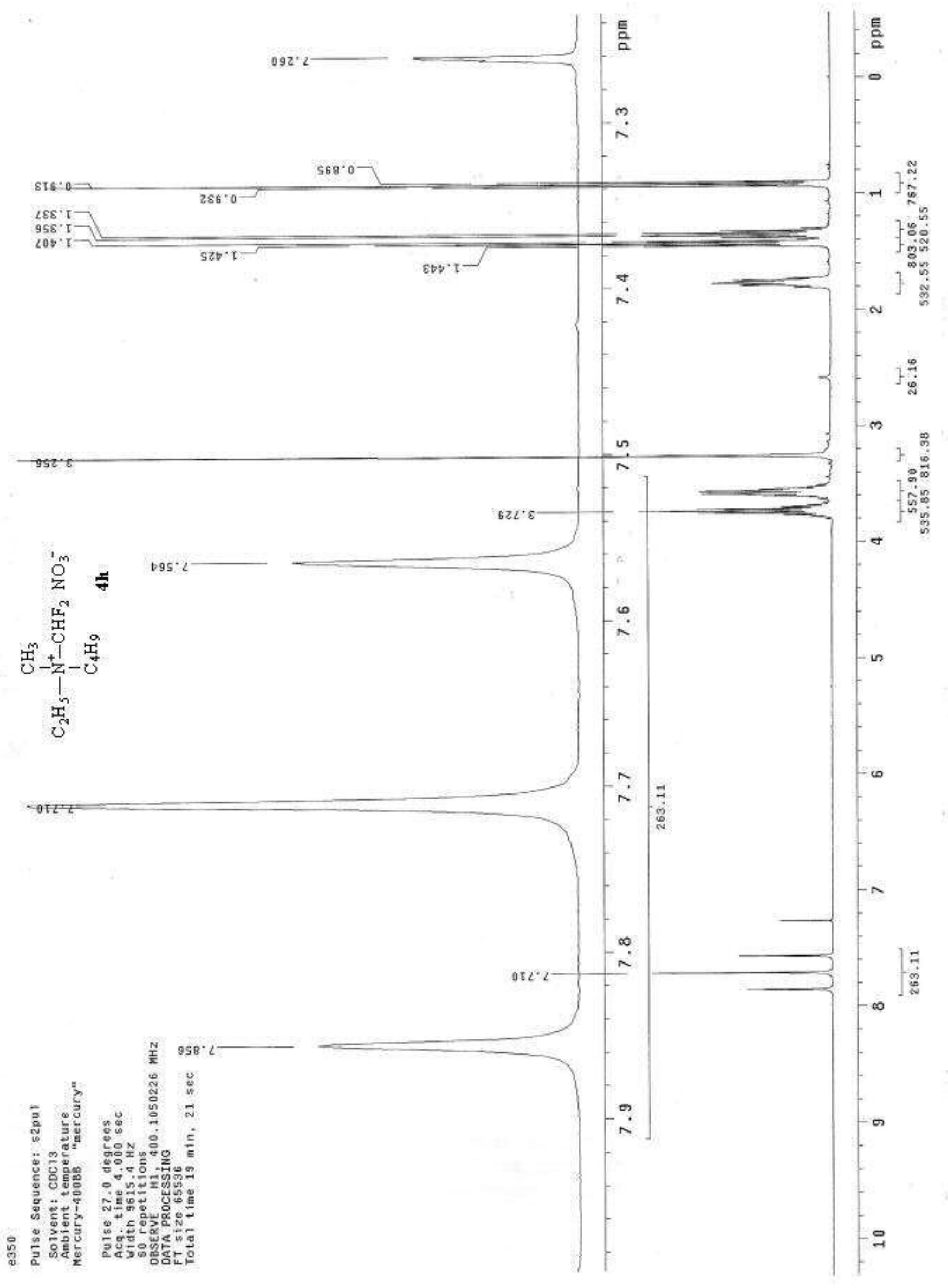




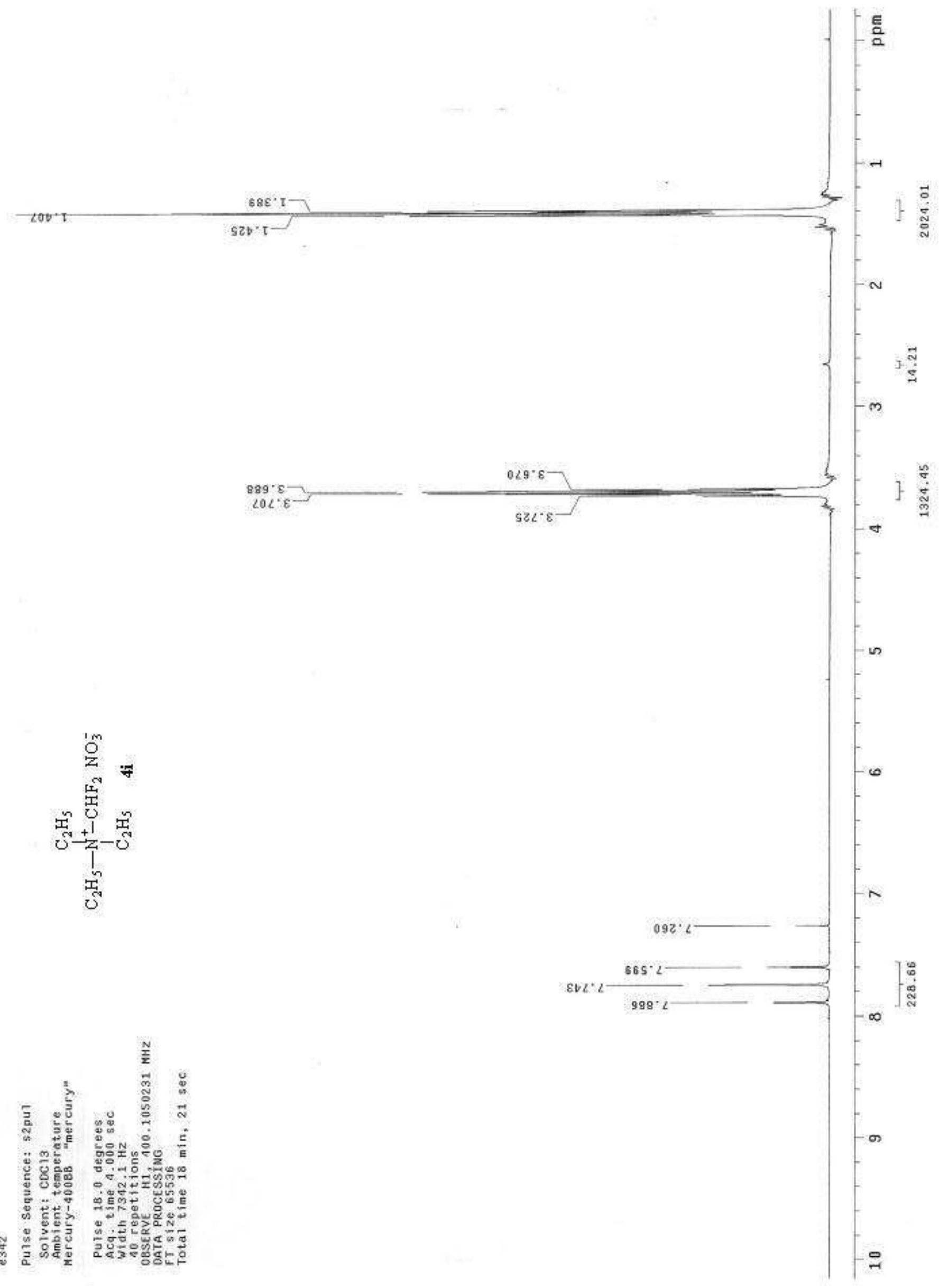




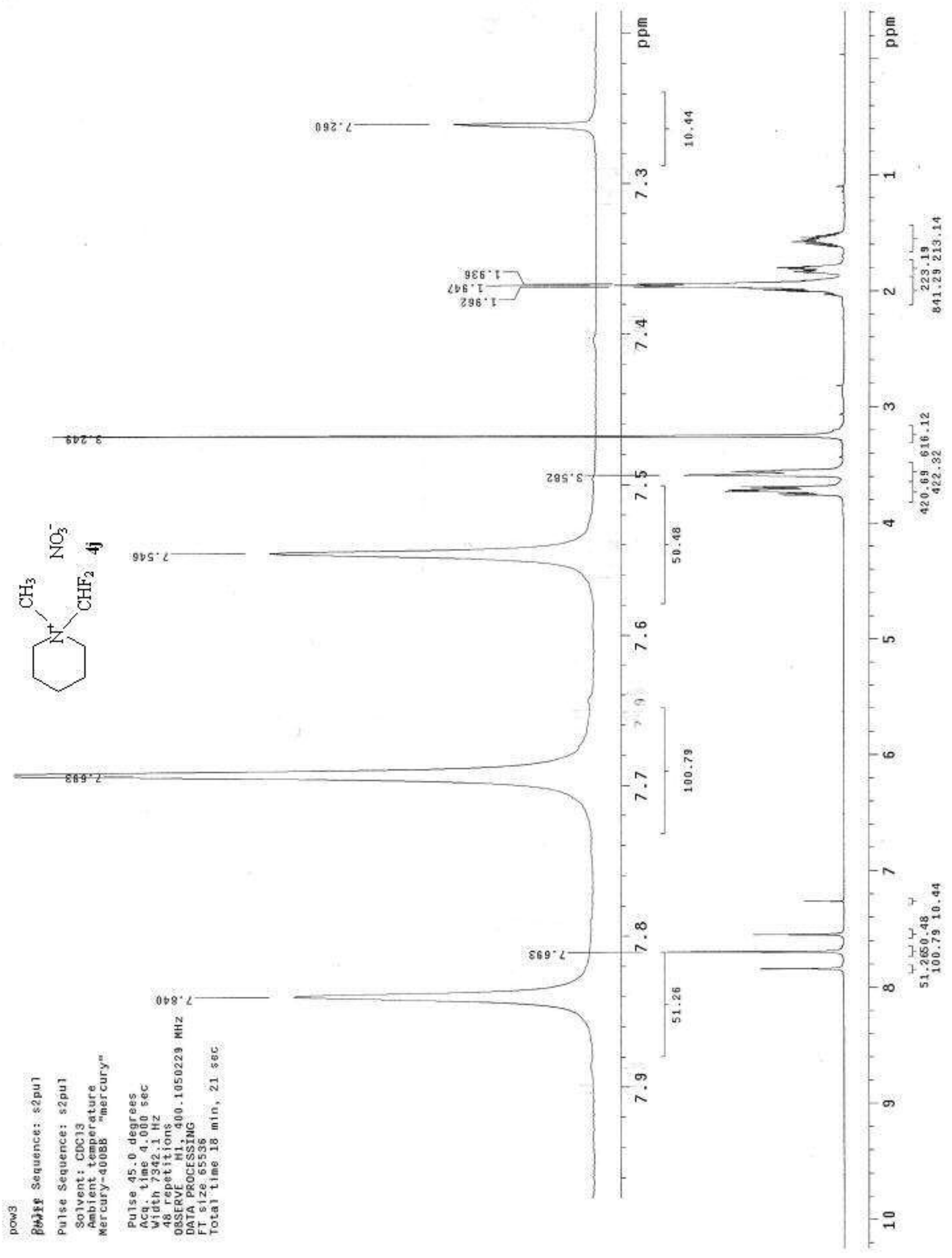




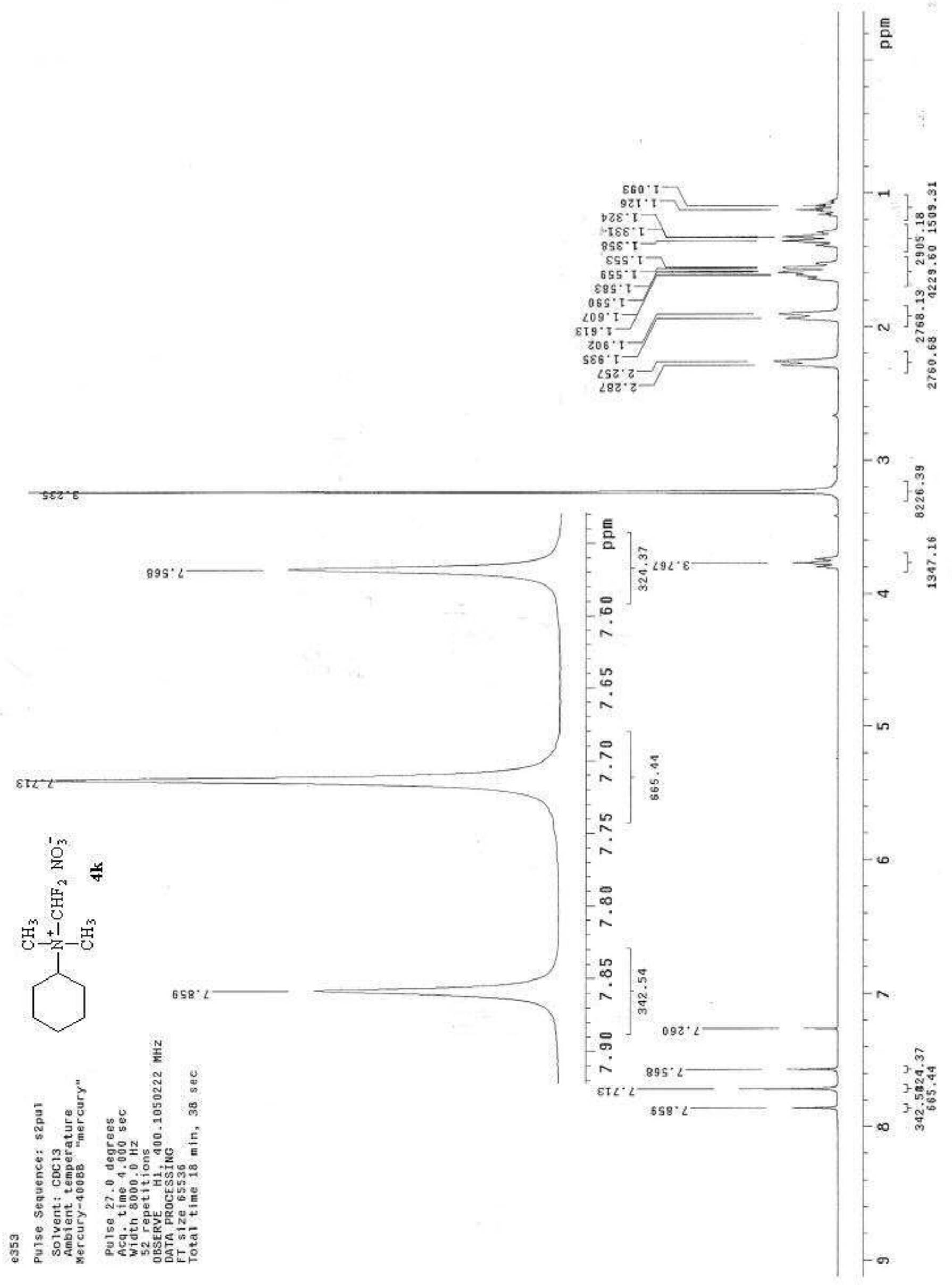




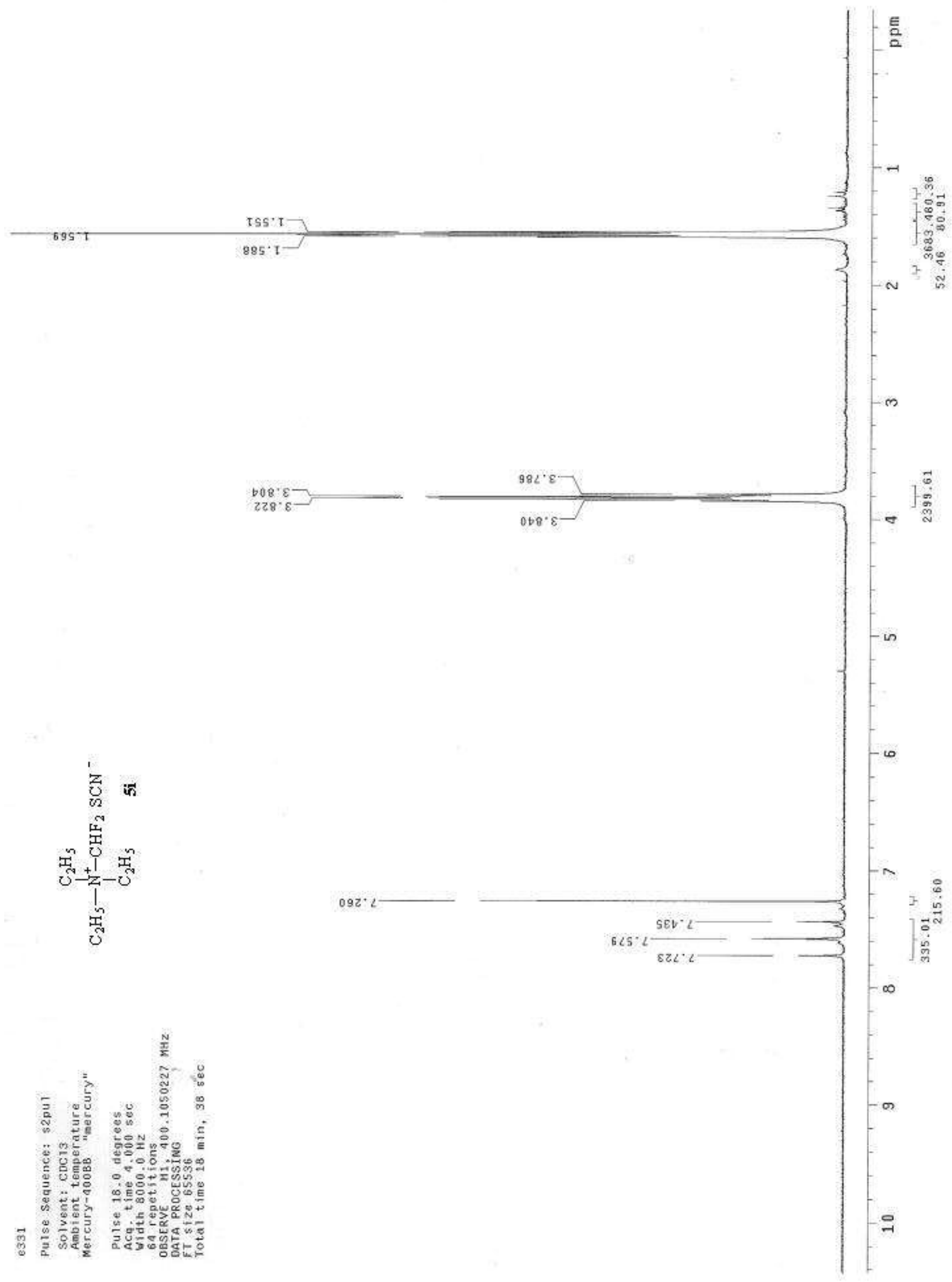




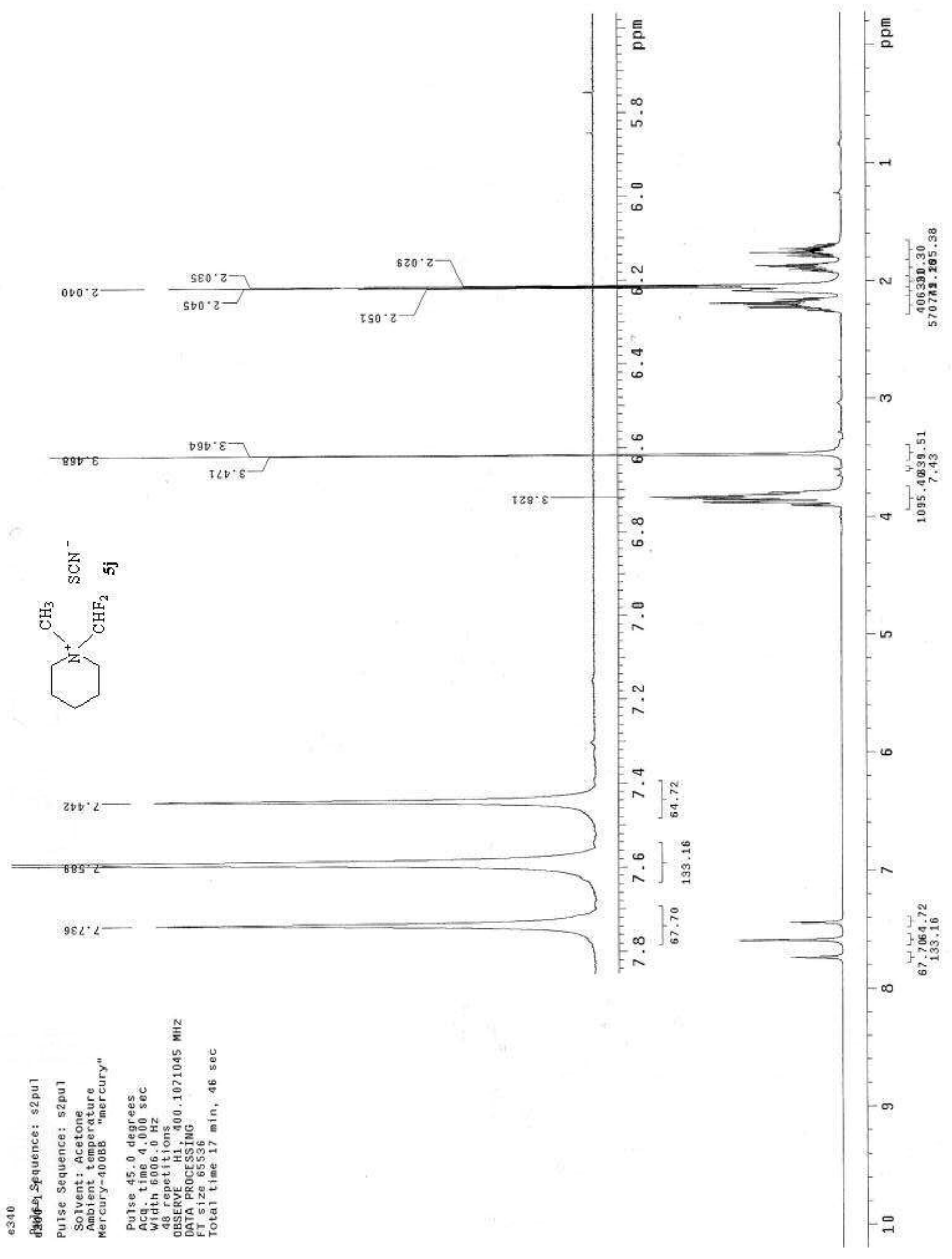

